\title{
Tonic and phasic alertness training: a novel behavioral therapy to improve spatial and non-spatial attention in patients with hemispatial neglect
}

\author{
Joseph M. DeGutis ${ }^{1 *}$ andThomas M. Van Vleet ${ }^{2}$ \\ Geriatric Research Education Clinical Center, Boston VA Healthcare System, Boston, MA, USA \\ 2 Northern California VA Healthcare System, Martinez, CA, USA
}

\section{Edited by:}

Donald T. Stuss, Baycrest Centre for Geriatric Care, Canada; University of Toronto, Canada

Reviewed by:

Ian Robertson, Trinity College, Ireland

Sam Gilbert, University College

London, UK

*Correspondence:

Joseph M. DeGutis, VA Boston Healthcare System (182JP), $150 \mathrm{~S}$

Huntington Ave., Boston, MA 02130, USA.

e-mail:degutis@wjh.harvard.edu
Hemispatial neglect is a debilitating disorder marked by a constellation of spatial and non-spatial attention deficits. Patients' alertness deficits have shown to interact with lateralized attention processes and correspondingly, improving tonic/general alertness as well as phasic/momentto-moment alertness has shown to ameliorate spatial bias. However, improvements are often short-lived and inconsistent across tasks and patients. In an attempt to more effectively activate alertness mechanisms by exercising both tonic and phasic alertness, we employed a novel version of a continuous performance task (tonic and phasic alertness training, TAPAT). Using a between-subjects longitudinal design and employing sensitive outcome measures of spatial and non-spatial attention, we compared the effects of 9 days ofTAPAT ( $36 \mathrm{~min} /$ day) in a group of patients with chronic neglect $(N=12)$ with a control group of chronic neglect patients $(N=12)$ who simply waited during the same training period. Compared to the control group, the group trained on TAPAT significantly improved on both spatial and non-spatial measures of attention with many patients failing to exhibit a lateralized attention bias at the end of training. TAPAT was effective for patients with a range of behavioral profiles and lesions, suggesting that its effectiveness may rely on distributed or lower-level attention mechanisms that are largely intact in patients with neglect. In a follow-up experiment, to determine if TAPAT is more effective in improving spatial attention than an active treatment that directly trains spatial attention, we trained three chronic neglect patients on both TAPAT and search training. In all three patients, TAPAT training was more effective in improving spatial attention than search training suggesting that, in chronic neglect, training alertness is a more effective treatment approach than directly training spatial attention.

Keywords: attention, visual, rehabilitation, neurological disorders, hemispatial neglect

\section{INTRODUCTION}

The neglect syndrome is a common consequence of lateralized brain injury in which patients fail to respond to stimuli on the side of space opposite their lesion (Buxbaum et al., 2004; Bartolomeo, 2007; Adair and Barrett, 2008). Neglect patients present with a wide array of spatial symptoms that may include deficits in attending to one's body (Beschin and Robertson, 1997), peri- and extrapersonal space (Bisiach et al., 1986; Halligan and Marshall, 1991), and parts of objects (Young et al., 1992). More recently, researchers have examined neglect patients' non-spatial impairments which may include deficits in sustaining attention (Hjaltason et al., 1996; Robertson et al., 1997b) and in the moment-to-moment engagement and disengagement of attention (Husain et al., 1997). These non-spatial impairments have been shown to modulate patients' spatial deficits and paradoxically, may be more fundamental to the neglect syndrome than patients' spatial symptoms (Lazar et al., 2002; Husain and Rorden, 2003). Together, patients' non-spatial and spatial deficits combine to make neglect an extremely disabling disorder. In fact, compared to patients with similar stroke pathology and other forms of cognitive impairment, patients with neglect score significantly lower on established measures of functional disability and activities of daily living (Katz et al., 1999). Though several therapies have been developed over the last 30 years to treat neglect, addressing the entire range of patients' symptoms and producing consistent improvements across patients remains a challenge.

One treatment approach that has shown promise in addressing a broad range of patients' symptoms are behavioral treatments that target tonic and phasic alertness deficits (Robertson et al., 1995; Thimm et al., 2006; for reviews of other treatments see Luaute et al., 2006; Pizzamiglio et al., 2006). Tonic alertness refers to intrinsic arousal that fluctuates on the order of minutes to hours. It is intimately involved in sustaining attention as well as provides the cognitive tone for performing more complicated functions such as working memory and executive control (Sturm et al., 1999; Posner, 2008). Functional MRI studies have shown that tonic alertness is supported by a supramodal network including predominantly right inferior frontal, inferior parietal, and anterior cingulate regions (Sturm and Willmes, 2001; Thiel et al., 2004) whereas neurophysiological studies have demonstrated the importance of the locus coeruleus (for a review, see Aston-Jones and Cohen, 2005). Patients with neglect commonly have lesions that involve this network and 
typically demonstrate low general arousal (Heilman et al., 1978), marked deficits in sustaining attention (Hjaltason et al., 1996; Robertson et al., 1997b), and a significant decrement in vigilance over time (Malhotra et al., 2009).

In contrast to tonic alertness, phasic alertness is the rapid change in attention due to a brief event and is the basis for operations such as orienting and selective attention (Husain and Rorden, 2003; Posner, 2008). Phasic alertness is typically studied with infrequent, unpredictable warning signals preceding the presentation of a stimulus (Robertson et al., 1998; Posner, 2008). The neural mechanisms supporting phasic alertness have shown to be highly overlapping with but more extensive than tonic alertness, extending to left frontal and parietal regions (Coull et al., 2001; Sturm and Willmes, 2001). Patients with neglect have been shown to have marked deficits in phasic alertness (Husain et al., 1997). For example, when detecting two targets embedded in a rapid serial presentation of characters presented at fixation [attentional blink (AB) task], Husain et al. (1997) found that neglect patients required a $1400 \mathrm{~ms}$ interval between targets to consistently identify the second target whereas unimpaired controls required only $400 \mathrm{~ms}$.

Tonic and phasic alertness deficits interact with patients' spatial deficits. For example, tonic and phasic deficits predict the overall severity and chronicity of patients' spatial symptoms, in many cases better than the level of impairment shown on measures of spatial attention (Robertson et al., 1998; Husain and Rorden, 2003). This suggests that alertness deficits are a fundamental aspect of the neglect disorder. Changes in tonic and phasic alertness have also shown to modulate patients' spatial deficits. For example, reducing tonic alertness in recovered neglect patients via administration of a sedative causes a re-emergence of spatial neglect symptoms (Lazar et al., 2002). Additionally, boosts in phasic alertness, through unexpected tones, have shown to transiently improve patients' spatial neglect symptoms (Robertson et al., 1998). Modulation of spatial bias by alertness has also been demonstrated in unimpaired controls (Bellgrove et al., 2004; Manly et al., 2005), suggesting that this interaction is a general mechanism of attention rather than specific to the neglect disorder. Though the mechanism of the interaction between alertness and spatial attention is poorly characterized, it may be that enhanced alertness provides greater input to right dorsal front-parietal regions involved in the allocation of spatial attention, enabling these regions to better compete with left dorsal fronto-parietal regions (Corbetta et al., 2005).

Inspired by these findings, several labs have experimented with behavioral therapies to improve neglect based on the idea that prolonged and repeated stimulation of alertness mechanisms will produce a more alert attentional state and lead to general improvements in patients' non-spatial and spatial symptoms (Robertson et al., 1995; Thimm et al., 2006). Thimm et al. (2006) examined this hypothesis by engaging neglect patients in a stimulating computer game for a prolonged period of time each day to improve tonic alertness. After completing 3 weeks of training, patients improved their overall alertness and 6/7 patients improved on at least one of the standard spatial neglect tests administered. Still others have shown that teaching patients with neglect to increase their alertness through periodic self instruction can improve sustained attention as well as performance on a broad range of spatial attention measures (Robertson et al., 1995).
Building on the promise of these studies, we created a task that we reasoned would engage both tonic and phasic alertness components concurrently by requiring patients to frequently respond to non-target stimuli and inhibit their response to infrequent, temporally unpredictable target stimuli (tonic and phasic alertness training, TAPAT). The repetitive nature of responding to most stimuli requires endogenous alertness to avoid lapses of attention (Smallwood et al., 2004) whereas the infrequent, unpredictable inhibitory aspect of the task makes the target stimuli particularly salient and likely produces a strong phasic signal that alerts patients to stop the execution of their pre-potent response (Aron et al., 2004). We had patients perform this training procedure for $36 \mathrm{~min} /$ day over 9 days to promote patients sustaining benefits beyond the training session. To capture training-related improvements, we employed well-validated computer-based measures of spatial search (conjunction search) and object-based attention (landmark task). These have been shown to be more sensitive than standard paper and pencil measures of neglect, allowing us to reliably detect and quantify potentially clinically significant changes in symptoms (List et al., 2008). We also examined their performance on a challenging, speeded selective attentional task presented at central fixation (AB task; Shapiro et al., 1994). We also sought to quantify how individual differences in improvement on the training task, such as improvements in the ability to correctly inhibit responses to targets, were related to improvements on outcome measures.

In a follow-up experiment, using a within-subjects design with three chronic neglect patients, we compared TAPAT training to a control training procedure in which patients searched for missing objects among scenes for the same amount of time each day. Comparing these two treatments in the same patients allowed us to explore if training tonic and phasic alertness is more effective than having patients perform a supervised computer-based task directly targeting their visual search deficits.

\section{EXPERIMENT 1 \\ PATIENTS}

Twenty-four patients with chronic neglect symptoms (seven women) gave informed consent before participation, in compliance with the Institutional Review Boards of the VA Northern California Health Care System in Martinez, California and the VA Boston Healthcare System (see Table 1 and Figure 1). Patients were recruited on the basis of (1) right-sided lesion to the cerebral cortex, basal ganglia, or thalamus, (2) a demonstration of significant hemispatial neglect on two or more subtests on a clinical battery of tests for neglect (Standard Comprehensive Assessment of Neglect, McGlinchey-Berroth et al., 1996), and (3) normal or corrected vision that would allow them to see the stimuli on the computer screen. Patients with visual field defects in addition to hemispatial neglect were included as long as they fit the criteria above. For these four patients, care was taken to point out the spatial margins of the testing field prior to the start of each task. Patients' ages spanned 23-78 years (Mean of experimental group $=57, \mathrm{SD}=18.5$; Mean of control group $=66, \mathrm{SD}=9.0$ ), time since brain injury ranged from 4 months to 20 years (Mean of experimental group $=30.7$, $\mathrm{SD}=25.8$; Mean of control group $=44.3, \mathrm{SD}=67.6)$ and all were right-handed. 
Table 1 | Profiles of patients in the tonic and phasic alertness training (TAPAT) group and control group.

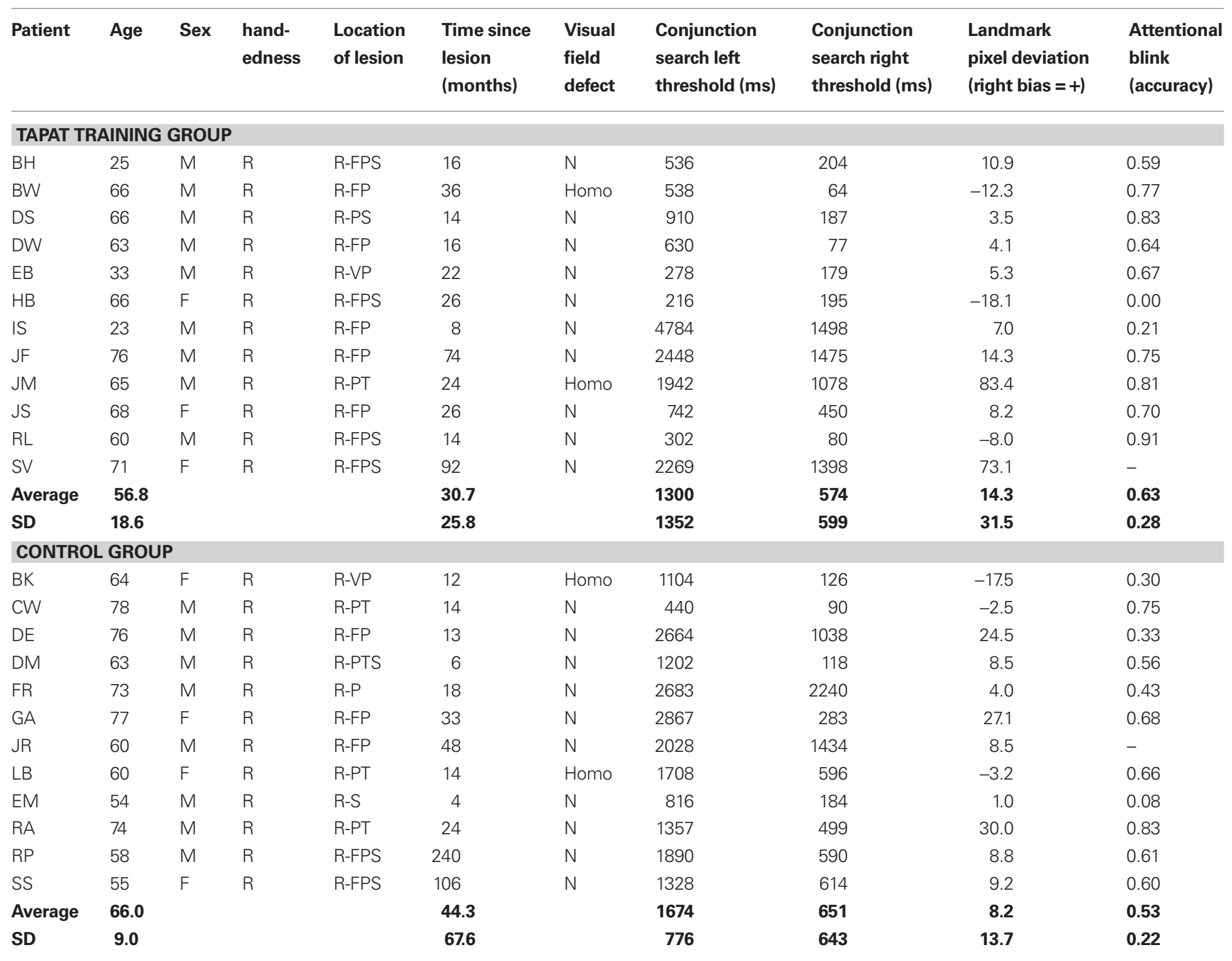

For the location of lesion, $R=$ right, $F=$ frontal, $P=$ parietal, $T=$ temporal, $S=$ subcortical, which includes thalamus, basal ganglia, or white matter. For visual field defect, homo = homonymous hemianopsia.

\section{STUDY DESIGN}

A between-groups AB design was used in which experimental and control groups were assessed on attention measures 1 day before and 1 day after either 9 days of TAPAT training or a 9-day wait period. To measure carryover effects from TAPAT training, patients in the TAPAT training group were also assessed 14 and 28 days after the completion of training.

Initially, four patients were run through TAPAT training (BW, DS, IS, and SV) before the control group was populated. We considered this initial group as proof of principle that TAPAT was effective and proceeded with randomizing subsequent patients to either TAPAT or the control condition. We randomly assigned patients to either TAPAT or the control condition until we reached 12 subjects in the TAPAT condition. At this point, there were seven patients in the control condition and the next five patients (DE, DM, LB, $\mathrm{RB}$, and SS) were assigned to the control condition to have an $n$ equivalent to the TAPAT condition. Though this does not qualify as a randomized design (Schulz and Grimes, 2002), the results suggest that the experimental and control groups were well matched on several variables including age, gender, lesion location, time since brain injury, and assessment performance (see Control Group vs. TAPAT Training Group results below).

\section{APPARATUS}

All assessments and training tasks were presented on a widescreen LCD panel $(33 \mathrm{~cm} \times 21 \mathrm{~cm})$ of a laptop computer. Patients viewed stimuli from a distance of $60-70 \mathrm{~cm}$. During training, patients responded by pressing the spacebar with their right hand.

\section{TONIC AND PHASIC ALERTNESS TRAINING}

Tonic and phasic alertness training consisted of three rounds of a 12-min task in which numerous centrally presented scenes were briefly displayed and patients were required to respond via a button press when the image was a non-target and withhold from responding when the image was a pre-determined target (see Figure 2). The 9 target scenes and 972 non-target scenes consisted of photographs 


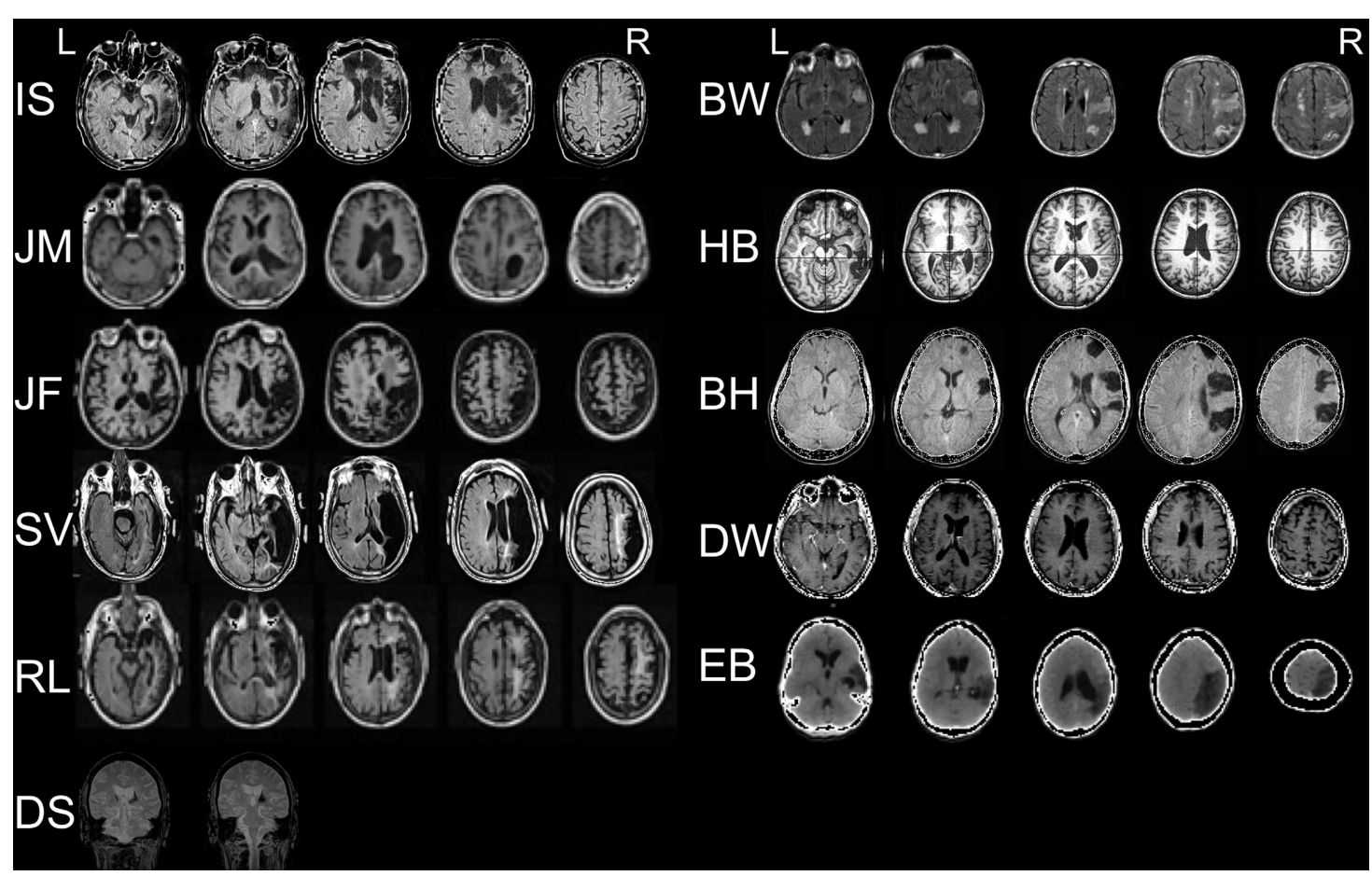

FIGURE 1 | MRI and CT scans of patients' lesions in neurological convention (excluding patient JS).

\section{Tonic and Phasic Alertness Training}

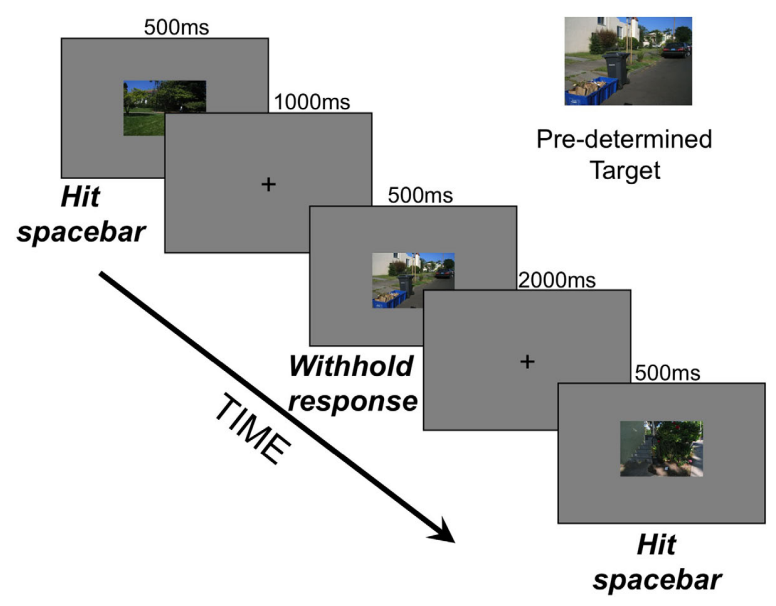

FIGURE 2 |TAPAT task. Each day, the training session began with the patients familiarizing themselves with a new target scene while reading the following instructions as the experimenter read them aloud: "You will see many scenes over the next $12 \mathrm{~min}$. Your job is to hit the spacebar as fast as you can for each scene except when the scene is the target scene. When you see the target scene, do not hit the spacebar. The target scene for today is the following [Example Target Scene]. Please take a minute to memorize this scene." Each 12-min round contained 360 trials: 36 target scenes (10\%) and 324 non-target scenes $(90 \%)$ randomly ordered. Each scene subtended a visual angle of $4^{\circ}$ (vertical) $\times 6^{\circ}$ (horizontal). On each trial, scenes were presented for $500 \mathrm{~ms}$ and between trials a blank screen with a fixation "+" at the center was shown for either 1000 ms (33.3\% of trials), 1500 ms (33.3\% of trials), or 2000 ms (33.3\% of trials). After completion of a round of 360 trials, patients took a short break (approximately $2 \mathrm{~min}$ ) before beginning the next 12-min round of 360 trials. Each day, patients performed three of these 12-min rounds for a total of 1080 trials. of a variety of common environments encountered within daily life (e.g., grocery store, kitchen, park). At the beginning of each day, the patient was presented with a new target scene, though the nontarget scenes remained the same each day. Target scenes had several distinguishing features that set them apart from non-target scenes (e.g., novel objects or novel color). The following are descriptions of the target scenes: Day 1 - rack of shoes at a department store, Day 2 - a door next to a bulletin board in a hallway, Day 3 - computers and electronic equipment on a desk, Day 4 - kayak in a sporting goods store, Day 5 - a fire hydrant outside an institutional building, Day 6 - mops and garbage cans in a room, Day 7 - shelves of laundry detergent at a grocery store, Day 8 - books shelving trucks in a library, and Day $9-$ a tree in a park. We measured commission accuracy, correct commission reaction time, and omission accuracy for every 120 trials, providing nine observations per daily session. This allowed us to calculate a mean and standard deviation for each measure on the first and final day of training and test for significant improvements for each patient.

It should be noted that the TAPAT training task is similar to a popular test of visual sustained attention, the sustained attention to response task (SART, Robertson et al., 1997a). For example, the response contingencies in TAPAT and SART are identical (respond to targets on $90 \%$ of the trials and withhold to non-targets $10 \%$ of trials). However, the timing varies considerably between the two tasks. TAPAT has a variable inter-trial interval with a trial occurring about every $2 \mathrm{~s}$ whereas SART has a consistent inter-trial interval and a trial about every second. The consistent inter-trial interval of the SART creates a situation where subjects are prone to lapses of attention and response anticipations (Smallwood et al., 2004). In contrast, the slower presentation and unpredictable appearance of each image in TAPAT likely, compared to the SART, promotes a 
more alert state and enhanced response control (Ryan et al., 2010). One final difference is that the SART task takes $5 \mathrm{~min}$ to complete whereas TAPAT takes $12 \mathrm{~min}$ to complete one round, with three rounds performed per session, for nine sessions. The longer-term practice with TAPAT likely produces different task strategies from SART, such as adopting a more cautious response strategy.

\section{COMPUTER-BASED NEUROPSYCHOLOGICAL ASSESSMENTS Conjunction search}

The conjunction search task requires searching for a target object amongst an array of 13 or 14 distracters that include same-colored objects and same-shaped objects (see Figure 4C and List et al., 2008 for a more complete description). Patients were instructed to fixate on the central crosshair at the start of each trial, and to indicate whether or not a target was present on each trial by verbally responding "yes" or "no." The experimenter entered patients' responses.

To determine the psychophysical threshold for each side of the display, we adopted a yes-no adaptive staircase procedure described by Kaernbach (1990). The initial display duration was set at $2000 \mathrm{~ms}$ and we manipulated the display duration to reach an adjusted accuracy rate of $75 \%$ (further details of this procedure are described by List et al., 2008). Staircases terminated after 10 reversals (when the answer from one trial to the next went from correct to incorrect or vice versa), and a threshold presentation time (TPT) was calculated by averaging the stimulus durations over the final six reversal points.

\section{Alternative conjunction search}

Before training, we administered the original conjunction search with the red square as the target. After training, to test whether conjunction search improvements generalize to a new target stimulus, we re-administered the original conjunction search task as well as an alternative version with a blue triangle as the target (see Figure 5).

\section{Landmark task}

The landmark task is a common test used to evaluate object-based attention in neglect (see Harvey et al., 1995). This task consisted of a single, black horizontal line that subtended $10^{\circ}$ of visual angle ( $5^{\circ}$ to the left and $5^{\circ}$ to the right of center) presented against a gray background (see Figure 6C). A red vertical reference line or landmark that subtended $0.125^{\circ}$ of visual angle above and below the horizontal bisected the line. For each trial, patients were instructed to first determine the center of the black line and to say whether the landmark is to the left or right of their subjective center of the line. There were two types of trials in which separate adaptive staircases were calculated: (1) one in which the landmark started from the left $\left(4^{\circ}\right.$ from center) and (2) one in which the landmark started from the right $\left(4^{\circ}\right.$ from center). Right starting trials and left starting trials were randomly intermixed.

If the patient reported that the landmark was to the right or left of center on a given trial, on the next trial of that type (right starting trial or left starting trial) the landmark was moved incrementally in the opposite direction. Reversals occurred when the patient changed their response for a given type of trial (i.e., went from saying "right of center" to saying "left of center" for a right starting trial). After every two reversals, the increments that the landmark was moved between trials decreased (reversals $1 \& 2-1.48^{\circ}$; reversals $3 \& 4-0$ $.74^{\circ}$; reversals $5 \& 6-0.37^{\circ}$; reversals $7 \& 8-0.15^{\circ}$; reversals $9 \&$ $\left.10-0.07^{\circ}\right)$. The task ended after 10 left starting trial reversals and 10 right starting trial reversals.

\section{Attentional blink task}

We utilized a conventional visual AB paradigm (Raymond et al., 1992; Shapiro et al., 1994) consisting of a rapid serial visual presentation of 14 items presented in the center of the screen (subtending $2^{\circ}$ of visual angle vertically and $1^{\circ}$ horizontally) with one or two target numbers embedded in 12 or 13 distracter letters (see Figure 7C, for a full description see Van Vleet and Robertson, 2006). Each character was presented on the screen for $120 \mathrm{~ms}$ with a $40 \mathrm{~ms}$ inter-stimulus interval. The first target number (T1) was white to maximize identification while the distracter letters and second target number (T2), when present, were black and more challenging to identify. T2 appeared either two positions after T1 $(200 \mathrm{~ms}$ after T1) or six positions after T1 (1040 ms after T1). T1 and T2 discrimination was a four alternative forced-choice judgment rather than a presence/absence judgment, preventing patients from conservative reporting of target detection when targets were not clearly attended. Patients verbally reported the identity of the targets and the experimenter coded responses via an external numeric keyboard. Only trials in which patients correctly identified T1 were used to calculate T2 accuracy.

\section{Data analysis}

To quantify TAPAT-related improvements in patients' performance, we compared assessment improvements in the TAPAT group (Post +1 minus Pre) to improvements in the Control group that waiting during the duration of the training period. Specifically, to quantify improvements on the conjunction search, we compared right and left thresholds at time points 1 and 2. Additionally, to obtain a single score for search bias for this task, we divided the left display duration by the right display duration. This search bias score was used in the impairment vs. improvement correlations as well as in the training strategy/improvement regressions (see below). To quantify improvements in the landmark task, we averaged the pixel deviation for left and right starting trials to create an average pixel deviation score. For the $\mathrm{AB}$ task, we quantified the overall percent correct of second target identification after correct first target identification and also split this up by the separate lags (2 or 6). To measure improvement on the TAPAT training task, we compared the mean of commission accuracy, correct commission reaction time, and omission accuracy on the first and last day of training.

To quantify the relationship between pre-training deficits and amount of improvement, we performed Pearson correlations between the pre-training scores and improvement scores (Post +1 minus Pre) for each assessment. Additionally, to determine how improvements on one assessment relate to improvements on the other assessments, we performed Pearson correlations between each improvement score, correcting for multiple comparisons using a false discovery rate of $p<0.05$. Lastly, to examine the relationship between improvements on components of the training task and assessment improvements (Post +1 minus Pre), we ran a stepwise linear regression for each assessment with omission accuracy, commission accuracy, and correct commission reaction time as predictors. 


\section{RESULTS}

\section{Improvement on TAPAT task in the training group}

Comparing performance on the first and final day of the training task shows that, as a group, there were no significant improvements on commission accuracy, correct commission reaction time, or omission accuracy (see Figure 3A). However, individual patients showed marked improvements on several different aspects of the task (see Figure 3B). This variability in patients' training task improvement patterns suggests that patients used different strategies to perform this task and allowed us to correlate improvements on the particular training components with the improvements on the assessments (see Predicting Assessment Improvements from Training Task Improvements).

\section{Control group vs. TAPAT training group}

At the first testing session there was no significant difference between the TAPAT training and control groups in age $[t(1,10)=1.61$, $p>0.13]$, time since brain injury $[t(1,10)=0.67, p>0.51]$, performance on the conjunction search (no significant main effect of group and no significant side by group interaction), landmark $[t(1,10)=0.60, p>0.56]$, or $\mathrm{AB}[t(1,9)=0.91, p>0.38]$. For the conjunction search, both TAPAT training and control groups were significantly slower at finding targets on the left compared to the right [TAPAT Group: $t(1,11)=2.94, p<0.05$; Control Group: $t(1,11)=5.76, p<0.01)]$. These results are consistent with previous results of neglect patients with right hemisphere lesions performing this task (List et al., 2008). For the landmark task, both groups only showed a trend toward a significant rightward deviation from the midpoint of the line [TAPAT Group: $t(1,11)=1.57, p>0.14$; Control Group: $t(1,10)=1.88, p>0.09$ ], likely due to the greater variability among subjects in this task.

To determine if TAPAT training had a larger effect than repeated testing over the same time period, for each measure we performed a repeated-measures ANOVA with pre/post as withinsubjects factors (for the conjunction search we also included side of display as a within-subjects factor) and TAPAT/Control as the between-group factor. Additionally, to reduce the contribution of general age-related decline in the speed of perceptual processing (for example, see Salthouse, 2000) to the results, we co-varied out patients' age.
A

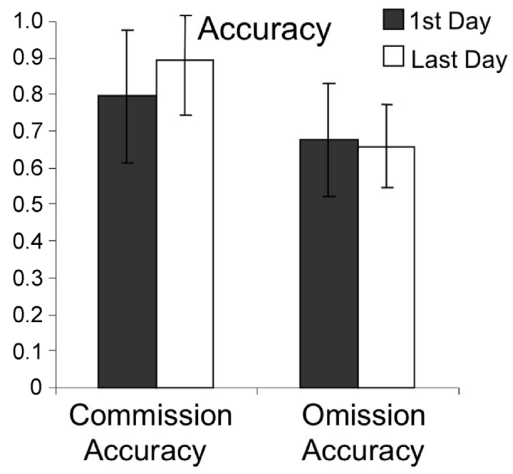

B

Individual Training Task Performance

\begin{tabular}{|c|c|c|c|}
\cline { 2 - 4 } \multicolumn{1}{c|}{} & CommACC & OmACC & CorComRT \\
\hline BH-pre & $95.8 \%$ & $51.4 \%$ & 492 \\
\hline post-pre & $-0.2 \%$ & $12.2 \%$ & -60 \\
\hline BW-pre & $98.6 \%$ & $57.4 \%$ & 569 \\
\hline post-pre & $-0.4 \%$ & $42.8 \%$ & 5 \\
\hline DS-pre & $33.4 \%$ & $94.4 \%$ & 746 \\
\hline post-pre & $2.0 \%$ & $0.0 \%$ & 24 \\
\hline DW-pre & $99.0 \%$ & $81.0 \%$ & 569 \\
\hline post-pre & $0.5 \%$ & $11.3 \%$ & -45 \\
\hline EB-pre & $98.5 \%$ & $22.2 \%$ & 331 \\
\hline post-pre & $1.5 \%$ & $-2.0 \%$ & -30 \\
\hline HB-pre & $96.8 \%$ & $64.8 \%$ & 556 \\
\hline post-pre & $-0.6 \%$ & $-9.6 \%$ & -40 \\
\hline
\end{tabular}

FIGURE 3 | Group (A) and individual (B) results of performance on the training task during the first and last day of training (error bars represent the standard error of the mean). There were no significant group changes with training $(\mathbf{A})$, though individuals demonstrated significant improvements and

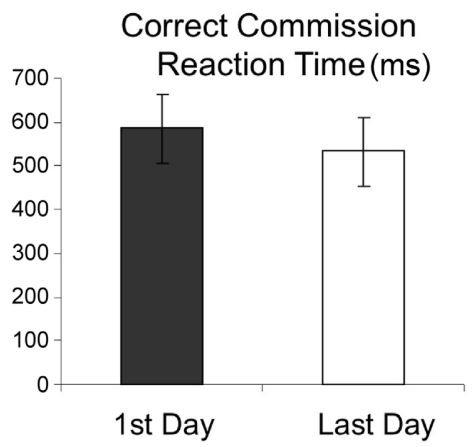

Significant Significant Individual Individual Improvement Decrement

\begin{tabular}{|c|c|c|c|}
\cline { 2 - 4 } \multicolumn{1}{c|}{} & ComACC & OmACC & CorComRT \\
\hline IS-pre & $75.7 \%$ & $55.1 \%$ & 640 \\
\hline post-pre & $18.1 \%$ & $14.7 \%$ & -139 \\
\hline JF-pre & $57.7 \%$ & $91.9 \%$ & 916 \\
\hline post-pre & $30.1 \%$ & $-9.6 \%$ & -142 \\
\hline JM-pre & $70.0 \%$ & $86.0 \%$ & 459 \\
\hline post-pre & $30.1 \%$ & $-5.9 \%$ & -56 \\
\hline JS-pre & $81.9 \%$ & $83.3 \%$ & 637 \\
\hline post-pre & $-2.2 \%$ & $-30.9 \%$ & -16 \\
\hline RL-pre & $70.8 \%$ & $63.0 \%$ & 590 \\
\hline post-pre & $18.2 \%$ & $-31.5 \%$ & -84 \\
\hline SV-pre & $76.4 \%$ & $62.4 \%$ & 513 \\
\hline post-pre & $16.2 \%$ & $-11.3 \%$ & 36 \\
\hline
\end{tabular}

decrements on different aspects of the training task (highlighted in gray and black). This was determined by performing Wilcoxon signed rank test on each component of training and assessing the calculated Z-score statistic at the $p<0.05$ level. 


\section{Conjunction search}

The side of display $($ left/right $) \times$ pre/post $\times$ TAPAT/Control analysis showed no significant main effects of side of display, pre/post, or TAPAT/Control, but revealed a significant 3-way interaction of side of display $\times$ pre/post $\times$ TAPAT/Control $[F(1,21)=4.588, p<0.05]$. This interaction was driven by greater improvements in searching the left side of the display after TAPAT training compared to the repeated testing session in the control group (see Figure 4). In fact, 1 day after TAPAT training, there was no significant difference between the TPT for the left and right side of the display [mean Left $=580 \mathrm{~ms}$ vs. Right $=400 \mathrm{~ms} ; t(10)=1.65, p>0.10]$. However, 13 days later, these improvements faded and the difference between detecting left and right targets was not significantly different from before training. The results of Post +28 days were not significantly different Post +14 days, showing a significantly greater threshold for left targets compared to right targets.

For the TAPAT group, the degree of impairment on the conjunction search before training (as measured by the left TPT divided by the right TPT) was highly correlated with the amount of improvement at Post $+1[r=0.94, t(10)=8.713, p<0.001]$, suggesting that patients with the greatest impairment on the conjunction search demonstrated the greatest improvement after training (see Figure 8A).

\section{Alternative conjunction search}

To assess whether, in the TAPAT training group, the effects of training generalize to searching for a novel target, these patients were assessed on an alternative color-shape conjunction search task at Times 2 and 3 (Post +1 Day and Post +14 Days). A $2 \times 2$ repeatedmeasures ANOVA with side of display (left/right) and Time $(2,3)$ as factors revealed a significant main effect of time $[F(1,9)=5.71$, $p<0.05]$, but not side of display $[F(1,9)=0.12, p>0.73]$, and a significant interaction of Side $\times$ Time interaction $[F(1,9)=7.30$, $p<0.05$; see Figure 5]. The nature of this interaction mirrored that of the practiced conjunction search. $T$-tests comparing left vs. right mean reversal values on the alternative conjunction search task at Post +1 Day showed that patients exhibited no difference in the detection of left vs. right targets following training [mean Left reversals $=816$ vs. Right $=618 ; t(9)=1.19, p=0.23$ ], but took significantly longer to detect Left targets than Right targets at Post +14 Days [mean Left reversals $=1238$ vs. Right $=259$; $t(9)=2.51, p<0.05]$.

Improvement on the alternative conjunction task was measured by subtracting the alternative conjunction search bias score (left TPT) $/$ (right TPT) at Post +1 from the search bias score before training in the original conjunction search task. We found that

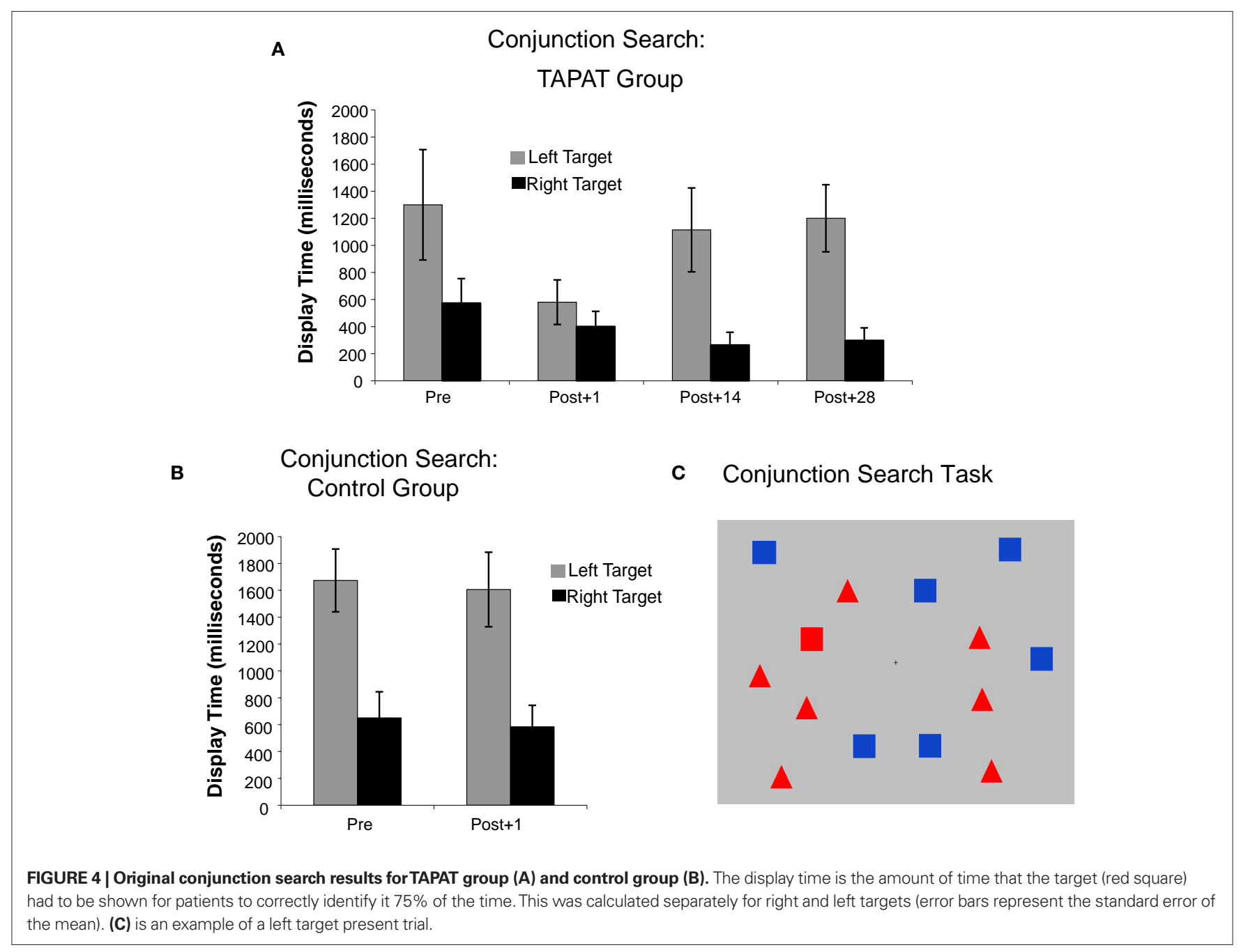




\section{Alternative Conjunction Search: TAPAT Group}
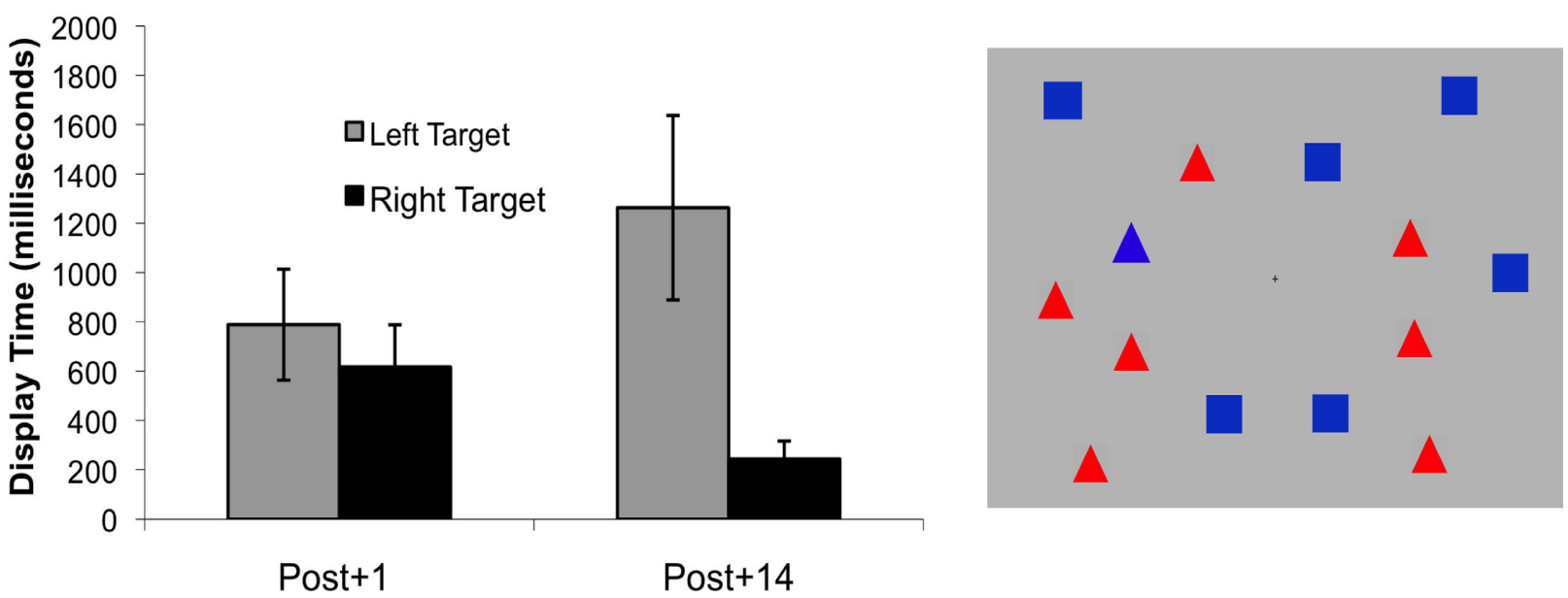

FIGURE 5 |Alternative conjunction search results forTAPAT group. The display time is the amount of time that the target (blue triangle) had to be shown for patients to correctly identify it $75 \%$ of the time. This was calculated separately for right and left targets (error bars represent the standard error of the mean).

this improvement score was highly correlated with the improvement score measured by the original conjunction search $[r=0.95$, $t(9)=8.605, p<0.001]$. Also, mirroring the results of the original conjunction search, the improvement score on the alternative conjunction search was highly correlated with the degree of pre-training impairment on the conjunction search task $[r=0.92$, $t(9)=6.64, p<0.001]$

\section{Landmark}

The repeated-measures Pre/Post $\times$ TAPAT/Control analysis of pixel deviation from center showed no significant main effects, but demonstrated a significant interaction $[F(1,21)=5.376, p<0.05]$, driven by a more leftward shift after training in the TAPAT group compared to repeated testing in the Control group (see Figure 6). For the TAPAT group, patients achieved a mean bias at Post +1 of close to 0 ( 0.45 pixels). At time points 3 and 4 , TAPAT patients shifted their estimation back to the right of the objective midpoint, significantly rightward of Time 2 ( $p s<0.05)$.

The degree of rightward deviation on the landmark task before training was highly correlated with the amount of improvement at Post $+1[r=0.81, t(10)=4.368, p<0.005]$, suggesting that patients with the greatest rightward deviation before training demonstrated the greatest leftward shift in their subjective center of the line (see Figure 8B).

\section{Attentional blink}

For the AB task, the repeated-measures Pre/Post $\times$ TAPAT/Control analysis on overall accuracy demonstrated a significant main effect of Pre/Post $[F(1,21)=18.83, p<0.005]$ as well as a significant Pre/Post $\times$ TAPAT/Control interaction $[F(1,21)=8.118, p<0.05]$. This interaction was driven by patients' greater improvement after TAPAT training compared to repeated testing in the Control group. In fact, after TAPAT training, patients performance was similar to unimpaired age-matched controls performing the identical task
(Van Vleet and Robertson, 2006). Splitting up the AB accuracy into the separate lags ( 2 and 6 ) and comparing performance before and after TAPAT training revealed a significant improvement for both lag 2 [Mean before training $=61 \%$, Mean Post +1 Day $=0.75 \%$; $t(9)=2.36, p<0.05$ ] as well as lag 6 [Mean before training $=64 \%$, Mean Post +1 Day $=0.88 \% ; t(9)=2.36, p<0.05]$. Consistent with the other measures, these improvements faded and failed to be significantly greater than pre-training by time points 3 and 4 $($ Post +14 and Post +28$)$.

The amount of accuracy improvement that patients demonstrated at Post +1 was highly correlated with severity of impairment before training for both lags $2[r=0.79, t(10)=3.866, p<0.005]$ and $6[r=0.81, t(10)=4.368, p<0.005]$. This suggests that patients with the worst performance on the $\mathrm{AB}$ showed the greatest improvements (see Figures 8B,C).

\section{Correlations between individual assessment improvements}

To determine the independence of training-related improvements on each assessment, we performed Pearson correlations correcting for multiple comparisons using a false discovery rate of $p<0.05$ (see Table 2). Only the correlation between the original and alternative conjunction search tasks survived this threshold, indicating that assessment improvements were largely independent of one another.

\section{Predicting assessment improvements from training task improvements}

To determine if improvements on components of the training relate to improvements on each assessment, we ran five separate stepwise linear regression analyses with change in omission accuracy, commission accuracy, and correct commission reaction time as predictors and the change in performance (from pre-training to Post + 1) on the conjunction search, alternative conjunction search, landmark, $\mathrm{AB}$ lag 2, and $\mathrm{AB}$ lag 6 as the dependent variables. 


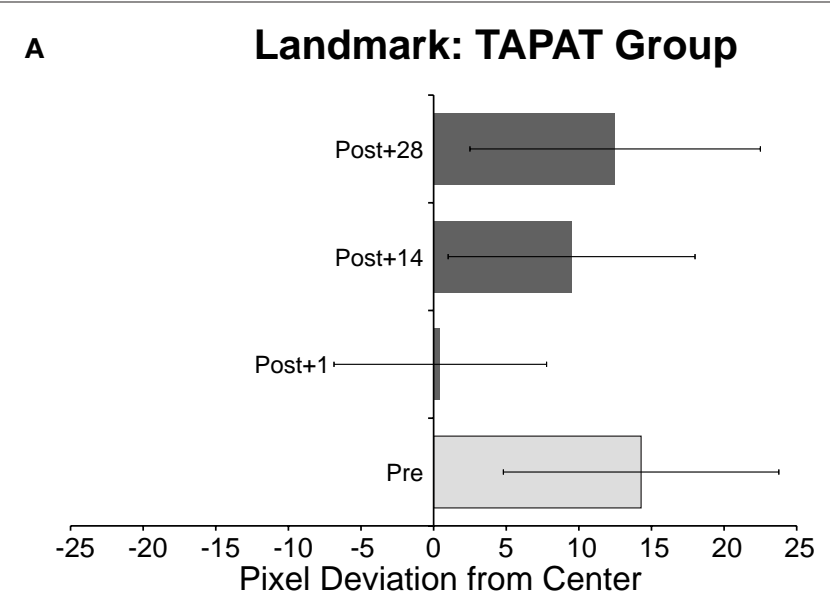

B Landmark: Control Group

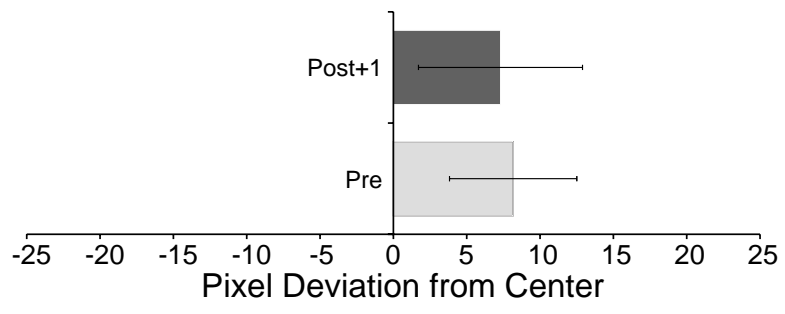

C

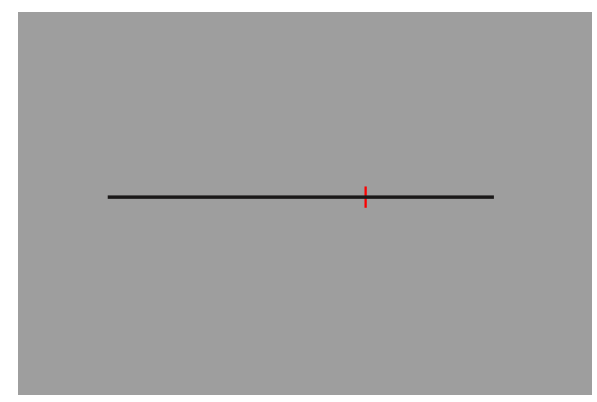

FIGURE 6 | Group landmark results forTAPAT (A) and Control (B) groups. The pixel deviation from center measures patients' subjective perception of the center of the black line compared to the actual center (left is negative, right is positive). Error bars represent the standard error of the mean. (C) is an example of a landmark display.

For the conjunction search model, we found that increased omission accuracy significantly predicted improvements on the conjunction search task $\left(R^{2}=0.372, p<0.05\right.$, see Figure 9B), while changes in the commission accuracy and reaction time did not predict improvement $(p=0.25$ and $p=0.49$, respectively). The alternative conjunction search model showed a similar trend as the original conjunction search with increased omission accuracy predicting improvement, but failed to reach significance $(p>0.06)$.

The landmark model showed a distinct pattern of results from the conjunction search tasks. Decreased correct commission reaction time significantly predicted improvement on the landmark task (change in deviation toward the left, $R^{2}=0.417, p<0.05$, see Figure 9C), whereas omission and commission accuracy did not significantly predict improvement $(p=0.17$ and $p=0.13$, respectively).

For the AB models, improvements in lag 2 accuracy was significantly predicted by commission accuracy $\left(R^{2}=0.396, p<0.05\right.$, see Figure 9D), but not omission accuracy $(p=0.18)$ or correct commission reaction time $(p=0.11)$. Improvements at lag 6 of the $\mathrm{AB}$ were not significantly predicted by improvements on any components of the training task.

\section{Post hoc analyses of training strategy and pre-training impairment on assessment improvements}

For assessments that showed that both pre-training impairment and training strategy significantly predicted improvement (CS, LM, $A B-2$, see Figures 8 and 9), we sought to measure the independence of these predictors. In particular, we sought to determine if the effects of training task strategy were mediated by level of pre-training impairment or vice versa. We ran mediation analyses and corrected for multiple comparisons using a false discovery rate of $p<0.05$. For performance on the conjunction search (CS), we performed mediation analyses of omission accuracy and pretraining conjunction search score on CS improvement. We found that pre-training CS impairment significantly mediated the effect of omission accuracy on conjunction search improvement (Sobel test statistic $=2.51, p=0.012$ ). There was only a trend of omission accuracy mediating the effect of pre-training CS impairment on conjunction search improvement (Sobel test statistic $=1.82$, $p=0.068)$. For the landmark task (LM) and AB tasks (AB, lag 2), we conducted mediation analyses of correct commission reaction time and correct commission accuracy, respectively, versus pretraining performance. We found no significant mediation effect of either predictor on the other (pre-training performance) for both tasks. Thus, training strategy had an independent effect on improvement on these measures beyond its relationship with the pre-training deficit.

\section{Relation of improvements and improvement longevity to age, time since injury, and lesion location}

For each assessment, there was no significant correlation between improvement or improvement longevity and age, time post brain injury, or lesion location. Though this could suggest that training enhances lower-level neuromodulatory mechanisms that are not particularly sensitive to enhanced cortical plasticity (as found in younger and more acute patients), it more likely reflects the inadequate power to detect these effects in the current sample. Additional patients and high-resolution brain mapping would be useful to better characterize this potential relationship.

\section{EXPERIMENT 1 SUMMARY}

Experiment 1 demonstrates that 9 days of TAPAT for $36 \mathrm{~min} /$ day significantly improved spatial and non-spatial attention in 12 chronic neglect patients. Comparing these results to the Control group in which the outcome assessments were re-administered after the same time period as TAPAT clearly demonstrates that TAPAT-related improvements were not due to simple practice effects. The magnitude of the TAPAT-related benefits on spatial 
A

\section{Attentional Blink: TAPAT Group}

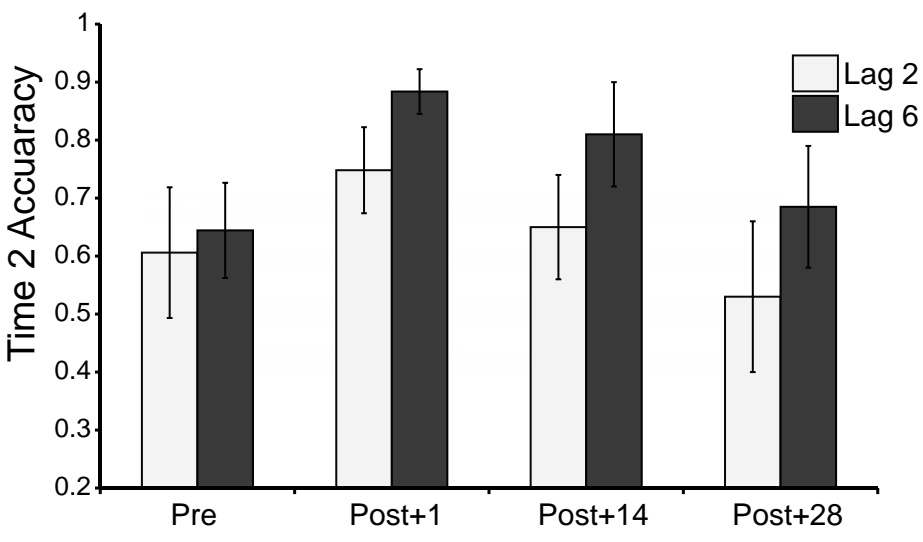

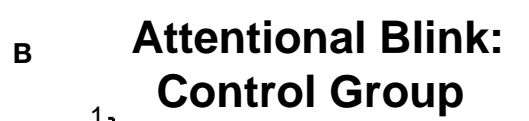

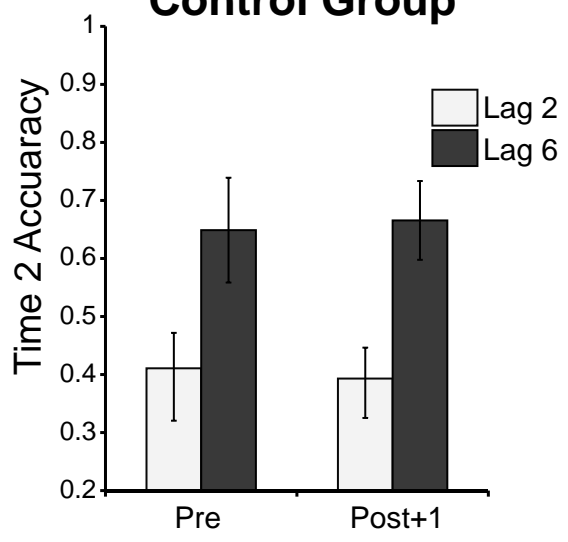

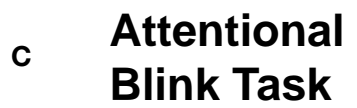

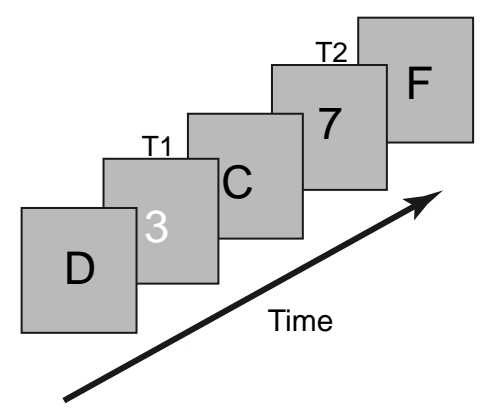

FIGURE 7 | Group attentional blink results forTAPAT (A) and Control (B) groups. Correct Time 2 discrimination accuracy is the percentage of correct identifications of the second target (in the example, 7) when correctly identifying the first target (in the example, 3). This is calculated for when the second target lags the first target by two positions (see example) or six positions. Error bars represent the standard error of the mean. (C) is an example of a lag 2 attentional blink trial: 3 is the first target (T1) and 7 is the second target (T2). attention are particularly striking given the fact that training was conducted at central fixation and did not directly train spatial attention. These results support the notion that training modulatory mechanisms, such as tonic and phasic alertness, can help support deficient processes such as search, midline estimation, and speeded selective attention.

The degree of patients' deficits on each measure strongly predicted the amount of patients' improvement, suggesting that TAPAT training particularly enhances performance on patients' most severely impaired domains. Interestingly, TAPAT-related improvements on one measure did not correlate with improvements on the other measures, suggesting that TAPAT influenced each attentional domain in an independent manner. Patients' improvements on components of the TAPAT training task also significantly predicted which assessments they improved on, though some of this effect could be explained by the fact that patients with particular impairments tended to adopt certain strategies on the training task and the impairment/improvement correlation drove this effect.
Though the effects of TAPAT were substantial and consistent across a heterogeneous group of chronic neglect patients, these effects faded 2 weeks after the completion of training. This is not entirely surprising given the limited time spent training (approximately $5 \mathrm{~h}$ ) and suggests that longer-term training may be necessary for sustained improvements. The positive side of this return to baseline is that it further confirms that TAPAT's effectiveness is not due to a general effect of practicing the assessments.

\section{EXPERIMENT 2}

Given these improvements in spatial attention following only 9-days of TAPAT training, we examined whether these effects are comparable to directly training spatial attention. Using a within-subjects design with three chronic neglect patients from Experiment 1, we compared TAPAT training to the same amount of time spent performing a task in which patients were required to locate subtle changes between flickering scenes that subtended the entire computer display (search training). Similar paradigms have shown to engage focused spatial attention and volitional search 


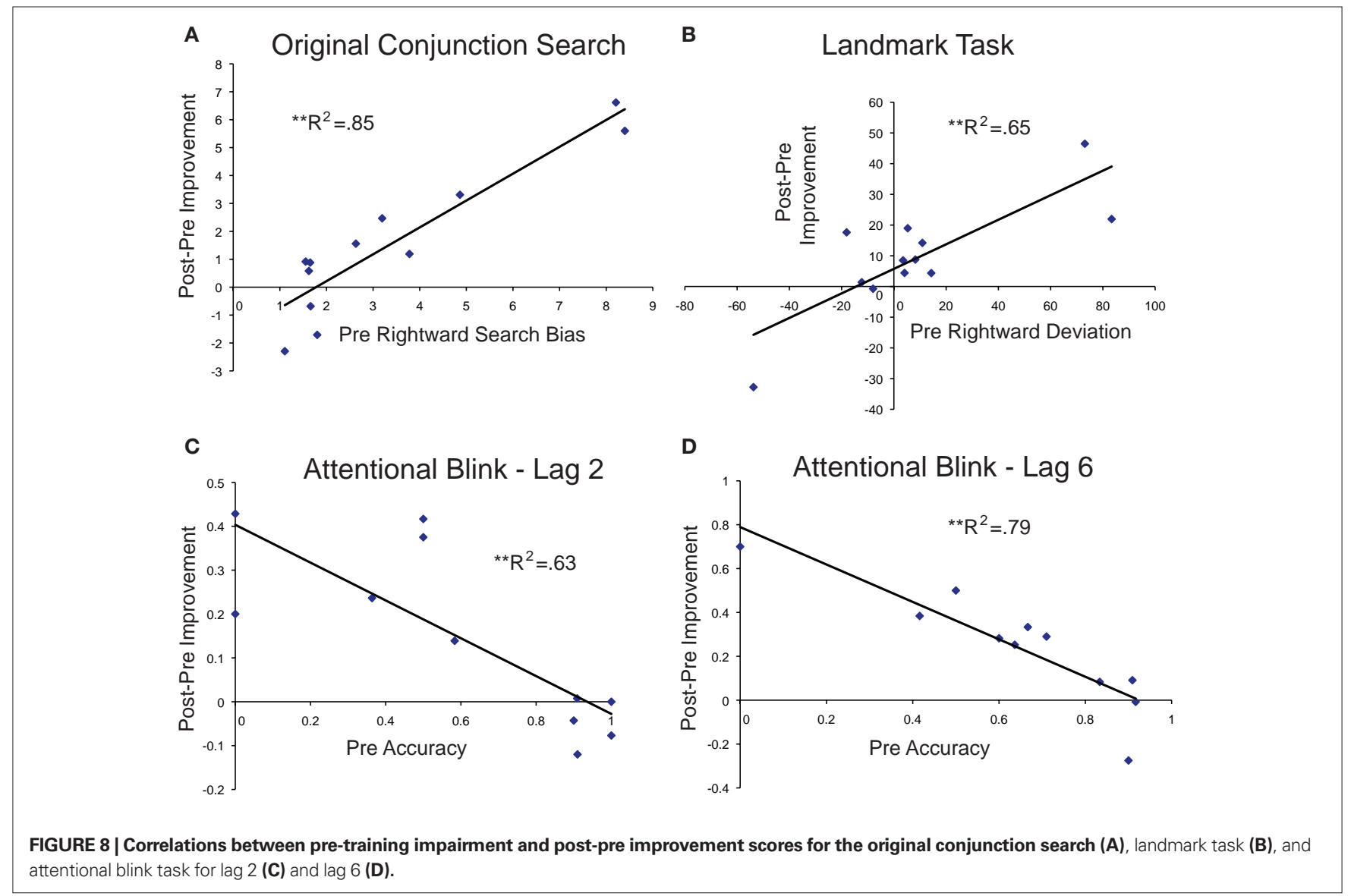

Table 2 | Pearson correlations between improvement scores on individual assessments.

\begin{tabular}{|c|c|c|c|c|}
\hline & $\begin{array}{l}\text { alternative } \\
\text { conjunction } \\
\text { search }\end{array}$ & landmark & $\begin{array}{l}\text { attentional } \\
\text { blink lag } 2\end{array}$ & $\begin{array}{l}\text { attentional } \\
\text { blink lag } 6\end{array}$ \\
\hline $\begin{array}{l}\text { conjunction } \\
\text { search }\end{array}$ & $0.95^{* *}$ & -0.39 & -0.09 & -0.40 \\
\hline $\begin{array}{l}\text { alternative } \\
\text { conjunction search }\end{array}$ & & -0.59 & 0.02 & -0.28 \\
\hline landmark & & & 0.35 & 0.30 \\
\hline $\begin{array}{l}\text { attentional blink } \\
\text { lag } 2\end{array}$ & & & & -0.21 \\
\hline
\end{tabular}

mechanisms (Rensink, 2002), processes that are consistently deficient in patients with neglect (for example, see Behrmann et al., 2004). The key differences between TAPAT and search training are that TAPAT engages sustained attention and phasic alertness to stimuli presented foveally (i.e., at central fixation without requiring eye movements) whereas search training targets spatial attention and requires patients to search via eye movements across a larger visual scene. Comparing these two training procedures in the same patients would allow us to explore if training alertness mechanisms is more effective than directly training patients' search impairments.

\section{PATIENTS}

Three patients with neglect symptoms from Experiment 1 (one woman) participated in Experiment 2 (BW, DS, and SV; see Table 1 and Figure 1 in white boxes for their lesions). These patients were selected based on their willingness to participate in a second treatment procedure and their ability to come to the laboratory for repeated training sessions. BW and DS's assessment score performance, time since lesion, and ages were within one standard deviation of the mean of the TAPAT training group. Though SV's age was similar to the TAPAT group, her time since lesion was substantially longer ( $>2 \mathrm{SD}$ ) and landmark score was more impaired than the mean of the TAPAT training group ( $>2 \mathrm{SD}$ ). In terms of response to TAPAT, DS and SV were within one standard deviation of the average improvement score for the conjunction search (left TPT/right TPT before - left TPT/right TPT after), whereas BW's conjunction search improvement was 1.6 standard deviations above the mean. For improvement on the landmark task, BW and DS were within one standard deviation of the mean improvement score, whereas SV showed an improvement 2.55 standard deviations above the mean.

\section{STUDY DESIGN}

A within-subjects repeated-measure design was used in which patients' spatial attention was assessed using the conjunction search and landmark tasks 1 day before and 1 day after 9-days of either TAPAT or search training. Performance of these two training programs was separated by approximately 3 months 
A

\begin{tabular}{|c|c|c|c|}
\hline & \multicolumn{3}{|c|}{$\begin{array}{l}\text { Improvement on } \\
\text { Components of TAPAT }\end{array}$} \\
\hline & $\begin{array}{c}\text { Omission } \\
\text { ACC }\end{array}$ & $\begin{array}{l}\text { Comm } \\
\text { ACC }\end{array}$ & \begin{tabular}{|c|} 
Correct \\
Comm RT
\end{tabular} \\
\hline Original cs & ** & -- & -- \\
\hline Alternative CS & -- & -- & -- \\
\hline Landmark & - & -- & ** \\
\hline$A B$ - lag2 & - & ** & - \\
\hline$A B-\operatorname{lag} 6$ & -- & -- & -- \\
\hline
\end{tabular}

C

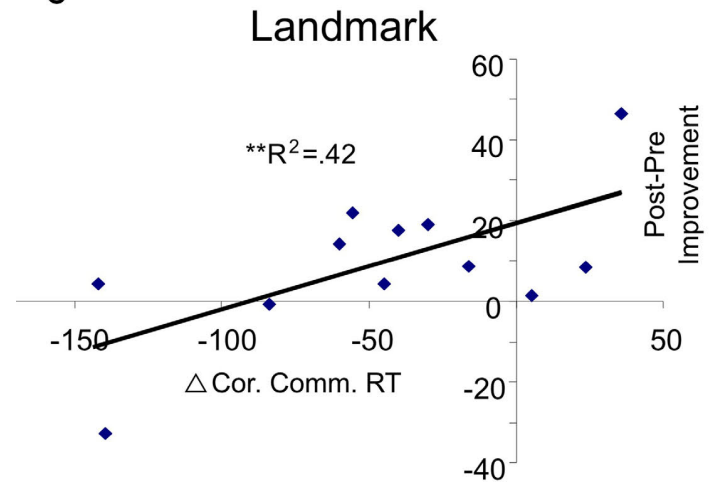

B Original Conjunction Search

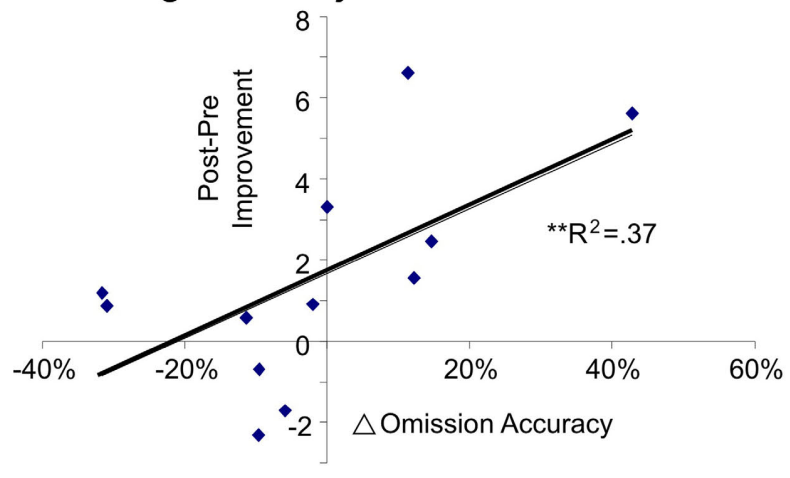

D

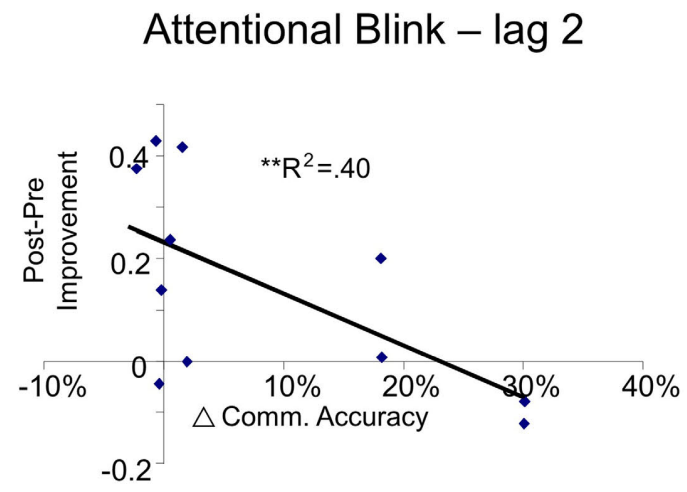

FIGURE 9 | Results of stepwise regressions predicting assessment improvement from training task improvement. (A) Shows the regression results predicting the post-pre improvement scores from improvements on components of the training task. ${ }^{*}$ indicates that the training component significantly predicted the particular assessment improvement ( $p<$.05). (B-D) Show plots of the assessments with significant improvement-training component relationships.

to ensure no carryover from the previous training program. SV performed TAPAT training first and search training second, whereas DS and BW performed the search training first and TAPAT training second.

\section{SEARCH TRAINING}

Search training consisted of a 36-min task in which patients performed visual change detection (for a review of these tasks, see Rensink, 2002). On each trial, two briefly presented (500 ms) real world scenes would alternate for $15 \mathrm{~s}$. These were full screen versions of the scenes used in TAPAT training. One scene would be intact and the other scene would be exactly the same but missing one object. Subjects were given the following instructions at the beginning of training: "You will be shown two flickering scenes for the next $15 \mathrm{~s}$. Your job is to find the missing object as fast as you can and tell the experimenter the identity of the missing object. There is a missing object on every trial so look around the entire scene until you find it." The experimenter would input patients' responses as correct or incorrect and, when the patient was ready, would start the next trial. To promote patients searching both sides of the display, half of the missing objects were on the left side of the display and half were on the right side of the display. Trials had six levels of difficulty which was based on reaction times to each scene in healthy control subjects $(N=5)$, ranking each scene based on reaction time, and separating the scenes into six bins.
Easier levels typically had very large object changes (e.g., person, car) and more difficult levels had small object changes (e.g., pen, toothbrush). The difficulty level was adjusted so that patients were approximately $75 \%$ correct at detecting the missing object on both sides of the display.

\section{RESULTS}

Because of the small number of subjects and because the likeliness that the data are not normally distributed, for each individual's data we used Wilcoxon signed ranks to compare performance before and after training (Wilcoxon, 1945). For the conjunction search task, we calculated the search bias (left display time/right display time) for each reversal at each time point, 1.0 indicating symmetrical searching and numbers $>1.0$ indicating a rightward bias (see Figure 10). Before training, all three patients showed longer search times on the left compared to the right and a search bias greater than 1.0. After TAPAT training, all three patients showed a significant reduction in the search bias score (SV: search bias before $=1.64$, after $=0.97, Z=2.201, p<0.05$; DS: search bias before $=5.43$, after $=1.58, Z=2.201, p<0.05$; BW: search bias before $=17.59$, after $=2.5, Z=2.201, p<0.05$ ) whereas after search training no patient showed a significant reduction in search bias while two patients actually showed a significant increase in search bias score. (SV: search bias before $=1.49$, after $=2.21, Z=-2.201$, $p<0.05$; DS: search bias before $=10.63$, after $=61.3, Z=-2.201$, 


\section{Conjunction Search}
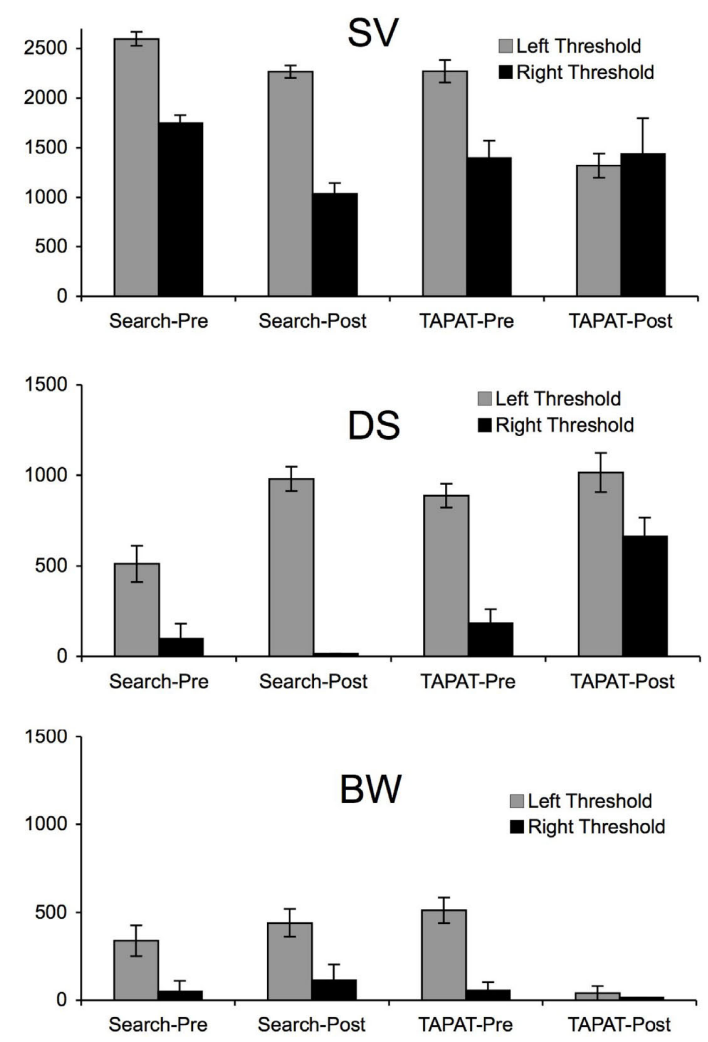

FIGURE 10 | Results of TAPAT training and search training in three chronic neglect patients for the conjunction search task and landmark task. Error bars represent the standard error of the mean. For the conjunction search, the display time is the amount of time that the target (red square) had to be shown
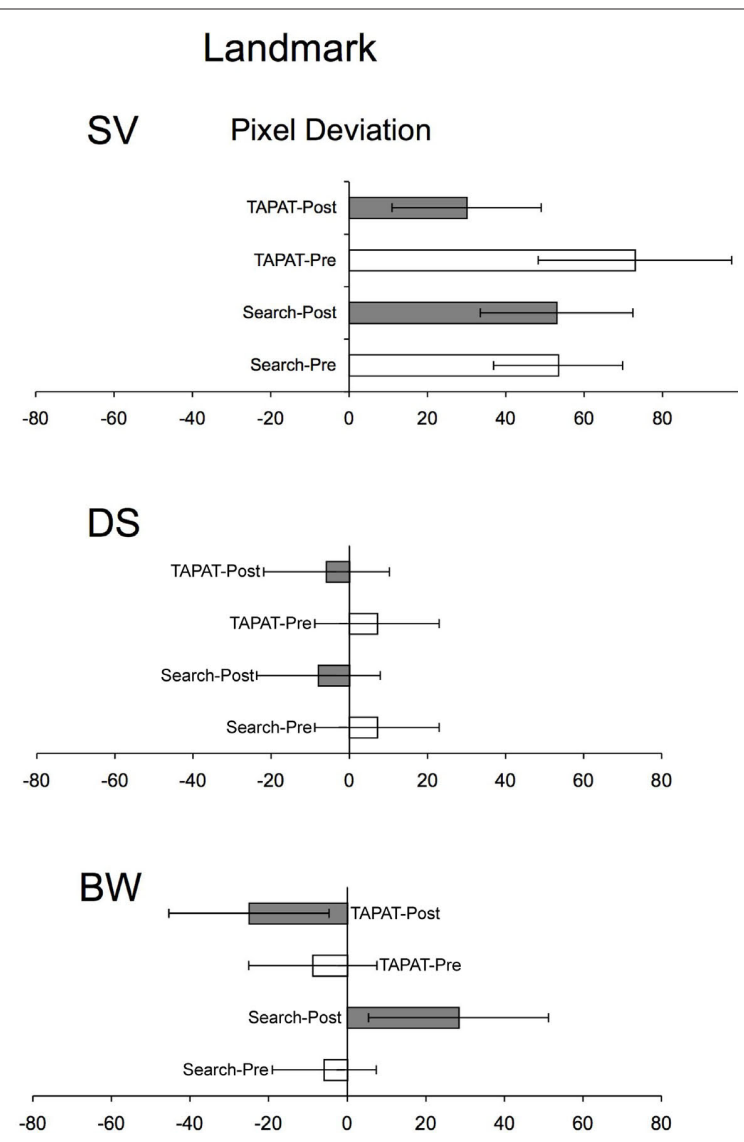

for patients to correctly identify it $75 \%$ of the time. This was calculated separately for right and left targets. For the landmark task, pixel deviation was collapsed across right starting and left starting trials to give an average pixel deviation to the right (positive values) or left (negative values). $p<0.05$; BW: search bias before $=13.21$, after $=11.39, Z=0.314$, $p>0.753)$. Remarkably, after TAPAT training, SV demonstrated a search bias very close to 1.0 , indicating a symmetrical search pattern.

A similar, though less pronounced pattern of results was found in the landmark task. Only one patient, SV, had a significant rightward bias on this task before training. After TAPAT training, SV showed a significant shift in her subjective midpoint toward the left (landmark bias before $=72.9$, after $=30.0, Z=2.226, p<0.05$ ), though she showed no significant change after search training (landmark bias before $=53.3$, after $=52.9, Z=0, p=1$ ). Though BW was not impaired on the landmark task before training, after TAPAT training he showed a trend toward shifting his subjective midpoint to the left (landmark bias before $=-8.8$, after $=-25.0$, $Z=1.897, p=0.058$ ) whereas after search training he significantly shifted his midpoint to the right. This indicates that, even though he was not impaired, TAPAT training further increased his relative awareness of the left side of the line. Patient DS significantly shifted his subjective midpoint to the left after both TAPAT (landmark bias before $=7.1$, after $=-5.8, Z=2.041, p<0.05)$ and search training (landmark bias before $=7.1$, after $=-7.9, Z=2.041, p<0.05$ ). These results suggest that TAPAT training shifted patients' subjec- tive midpoint to the left whether they were impaired or not. In contrast, search training had an inconsistent effect on patients' subjective midpoint estimation.

\section{EXPERIMENT 2 SUMMARY}

Though the results of experiment 2 are preliminary, they demonstrate that TAPAT training produced a more balanced pattern of spatial search and greater leftward shift in object-based attention than search training. This suggests that improvements after TAPAT training are likely due to training sustained attention and phasic alertness rather than simply engaging in a supervised computer-based training program. Further, these results are particularly striking because, unlike TAPAT, search training directly exercised patients' spatial attention mechanisms. This provides further evidence that neglect patients' fundamental deficits are with non-spatial processes (Robertson, 2001), and that these deficits must be remediated before higher-level spatial mechanisms. Though the results are consistent in these three patients, because neglect is such as heterogeneous disorder and these patients may not be typical of the greater population of neglect patients, a larger trial comparing TAPAT and search training is necessary to make stronger conclusions. 


\section{GENERAL DISCUSSION}

Together, Experiments 1 and 2 demonstrate that TAPAT significantly improves both spatial and non-spatial attention deficits in a broad range of chronic neglect patients. After training, we were unable to detect a rightward bias on sensitive tests of spatial search and midpoint estimation at the group level, suggesting that the effects of training were substantial. These significant training effects across a heterogeneous group of chronic neglect patients expands upon previous alertness training procedures that often demonstrate inconsistent improvements at the group level (for example see Sturm et al., 2004; Thimm et al., 2006). Because TAPAT training improved deficits in a group of patients with such a broad range of behavioral profiles and lesions, it suggests that TAPAT enhances attentional mechanisms that are largely intact in the majority of patients suffering from neglect.

The failure to find a lateralized search bias on the conjunction search task following TAPAT training is particularly striking given the fact that the conjunction search task is very sensitive to the presence of neglect, significantly more sensitive than standard paper and pencil measures (List et al., 2008). Notably, patients achieved this balance in search efficiency (left vs. right) after TAPAT training without sacrificing their level of performance on the right side of the display. This effect was replicated in the alternative conjunction search task initially run after training, demonstrating that improvements generalized to an unfamiliar search task. Restoring efficient, non-biased search on such a sensitive task suggests that the effects of training may be clinically relevant.

In addition to improvements in search after training, at the group level patients also improved their midpoint estimation on the landmark task. The landmark task is similar to the classic line bisection task, but it removes the motor component and is thought to reflect more perceptual processes (Harvey et al., 1995; Olk et al., 2004; Toraldo et al., 2004). This task can be solved using a variety of strategies, such as comparing the two sides of the bisected line or a more gestalt method of judging the center of the line. In an attempt to constrain the strategy patients employed, we instructed patients to judge whether the bisector was located to the right or left of center, invoking a more gestalt approach (Fink et al., 2002). Consistent with previous studies, patients showed a significant rightward bias before training (see Figure 6). After training, 11/12 patients moved their subjective center toward the left and as a group achieved a mean bias that approximated the objective center of the line.

The results on the $\mathrm{AB}$ task demonstrate that, in addition to improvements in spatial attention, training also produced significant group improvements in non-spatial, time-challenged, selective attention. While the $\mathrm{AB}$ task has elements similar to the training task such as central serial presentation and target detection, the considerably faster presentation of stimuli in the $\mathrm{AB}$ task put greater demands on updating visual working memory. Also, unlike the training task, the $\mathrm{AB}$ taxes patients' ability to rapidly disengage attention from the first target (T1) and re-engage attention to the second target (T2; Kranczioch et al., 2005). Consistent with prior studies, before training patients were impaired at detecting the second target at both early and later lags (Husain et al., 1997; Van Vleet and Robertson, 2006). After training, patients improved at detecting the second target at both lags 2 and 6 achieving a performance level comparable with a group of age-matched, neurologically intact participants (Van Vleet and Robertson, 2006). Improvements at each lag were not significantly correlated $(r=-0.21)$, suggesting that improvements were likely due to different mechanisms. For example, Lag 6 improvements may be due to a general improvement updating of visual working memory (Roth et al., 2006) and lag 2 improvements may depend more on the rapid engagement and disengagement of attention (Kranczioch et al., 2005).

A notable finding is that patients improved the most on outcome measures in which they showed the greatest pre-training impairment. This demonstrates that training can improve even patients' most severely impaired functions and suggests that the cognitive and neural architecture supporting these functions is remediable rather than permanently damaged. In addition, improvements on any one assessment were largely independent of improvements on other measures, demonstrating the individual nature of training-related improvements. This also suggests that improvement on one assessment is not mediated by improvement on the others. This is consistent with findings that show dissociations between visual search and object-based attention in neglect (for example, Ota et al., 2001). However, the finding that $\mathrm{AB}$ improvements are not related to search and landmark improvements is somewhat surprising considering that alertness modulates spatial bias (Robertson et al., 1998; Lazar et al., 2002) and the $A B$ is a direct reflection of alertness. It may be that the $\mathrm{AB}$ only reflects one aspect of alertness (phasic alertness) and that the interaction between alertness and spatial attention may rely more on other manifestations of alertness such as sustained attention.

In addition to a very strong impairment/improvement relationship for each assessment, improvements on specific aspects of the training task significantly predicted improvements on specific assessments. One explanation of this effect is that improved skills related to a specific training strategy bolstered mechanisms relevant to each particular assessment. An alternative explanation is that patients who were impaired on specific assessments were more apt to take a specific strategy on the training task and improve on that strategy. This explanation is consistent with the regression results showing that pre-training impairment scores were significantly better predictors of assessment improvement than training task strategies. For the conjunction search, pre-training impairment did significantly mediate the effect of omission accuracy on assessment improvement. However, pre-training impairment did not significantly mediate the effect of training strategy on assessment improvement for $\mathrm{LM}$ or $\mathrm{AB}$ lag 2, suggesting that for these measures training strategy had an independent effect on improvement beyond its relationship with pre-training deficits. Thus, for the $\mathrm{LM}$ and $\mathrm{AB}$ lag 2, improvements on components of the training task were significantly related to performance improvements. Studies with additional patients will be useful to better characterize the impact of training strategy on assessment improvement and its relationship to pre-training impairment.

Training-related improvements on the assessments lasted at least 1 day after completion of training, demonstrating that benefits persisted beyond the training session. One interpretation of why this effect persisted is that training stimulated neural circuits that remained active after training ended. This is consistent with repeated neural stimulation protocols, such as transcranial magnetic stimulation, producing therapeutic carryover effects that last for days beyond the stimulation session (Brighina et al., 2003; Naeser 
et al., 2005; Boggio et al., 2007). An alternative to this interpretation is that the carryover effects were due to enhancing cognitive skills. Supporting this idea, improvements on components of the training task were significantly correlated with improvements on the outcome measures, particularly for the landmark task and AB lag 2. This suggests that improved cognitive skills from training such as enhanced focused attention, sustained attention, and inhibition were recruited beyond the training session and generalized to performance on the outcome measures. It may be that these skills were not well-practiced enough to persist for 14 days after the completion of training and that similar to other training studies that produce more permanent effects (Brefczynski-Lewis et al., 2007; Draganski and May, 2008), more training may be necessary for longer-term improvements. Studies with additional patients, longer training durations, and more frequent post-testing sessions will be useful to further characterize the nature of TAPAT's carryover effects.

Why is TAPAT effective in improving patients' symptoms? Our hypothesis was that training tonic and phasic alertness together in one task would be more effective than methods focusing on tonic or phasic alertness alone (Robertson et al., 1995; Sturm et al., 2004; Thimm et al., 2006). This hypothesis was supported in that TAPAT training produced significant reductions in spatial bias at the group level and that all three components of the TAPAT training task were related to patients' improvements (see Figure 9). This suggests that TAPAT's effectiveness may be from concurrently engaging several aspects of non-spatial attention such as (1) endogenously sustaining alertness over an extended time and fighting lapses of attention (Smallwood et al., 2004) and (2) periodically engaging phasic alertness mechanisms in order to inhibit a pre-potent response to an infrequent target. Exercising these processes together may enhance non-spatial attention mechanisms more than focusing on either tonic or phasic alertness in isolation.

An alternative hypothesis is that TAPAT derives its effectiveness from training a more optimal attentional state (for a review, see Tang and Posner, 2009). For example, TAPAT may encourage an "exploitative" attention state as opposed to an "explorative" state (Aston-Jones and Cohen, 2005). According to Aston-Jones and Cohen (2005), the exploitative or phasic state is found when the current task is rewarding and is associated with a moderate level of arousal, the ability to rapidly engage and disengage attention, and enhanced working memory whereas the explorative state is found when the current task loses its rewarding value and requires seeking out alternative, more rewarding tasks. The exploitative state is associated with increased phasic responses in the locus coeruleus (LC), a brainstem nucleus intimately involved in alertness (AstonJones and Cohen, 2005). Since patients with neglect typically have low arousal and difficulties with engaging and disengaging atten-

\section{REFERENCES}

Adair, J. C., and Barrett, A. M. (2008). Spatial neglect: clinical and neuroscience review: a wealth of information on the poverty of spatial attention. Ann. N. Y. Acad. Sci. 1142, 21-43.

Aron, A. R., Robbins, T. W., and Poldrack, R. A. (2004). Inhibition and the right inferior frontal cortex. Trends Cogn. Sci. (Regul. Ed.) 8, 170-177.
Aston-Jones, G., and Cohen, J. D. (2005). An integrative theory of locus coeruleus-norepinephrine function: adaptive gain and optimal performance. Annu. Rev. Neurosci. 28, 403-450.

Bartolomeo, P. (2007). Visual neglect. Curr. Opin. Neurol. 20, 381-386.

Behrmann, M., Ebert, P., and Black, S. E. (2004). Hemispatial neglect and visual

tion, it may be that they rarely are in the exploitative/phasic state of attention. Several pieces of evidence suggest that TAPAT may work by enhancing the exploitative state of attention. First, TAPAT training may promote a moderate level of arousal by balancing stimulation, through constant responding to non-targets, with inhibition, through withholding a pre-potent response to targets. Also, improvements on the $\mathrm{AB}$, a task that has been correlated with $\mathrm{LC}$ phasic responses (Nieuwenhuis et al., 2005), suggests that TAPAT improves the engaging and disengaging of attention. Additionally, in monkeys, maximal phasic LC responses have been shown during tasks in which the monkey waits for a stimulus that is not predicted with much reliability and when the stimulus signals a cognitive or response-related shift (Bouret and Sara, 2005). This is a very close description to the TAPAT task, with the cognitive shift consisting of a shift in response contingency (i.e., rapid responding vs. effectively withholding response). Together, this suggests that TAPAT training may exercise an exploitative/phasic state of attention in neglect patients. Future studies will be useful to determine the plausibility of this mechanism.

\section{SUMMARY}

We demonstrate that 9 days of training on a continuous performance task that promotes tonic and phasic alertness significantly improves spatial and non-spatial attention in neglect. As a group, patients exhibited symmetrical spatial search (i.e., no rightward bias) and balanced object-based attention (i.e., mid line estimation consistent with objective midpoint) when examined $24 \mathrm{~h}$ post training, suggesting that TAPAT produced robust effects that lasted beyond the immediate training session. The results also show that improvement on several components of TAPAT training are predictive of behavioral improvements, suggesting that TAPAT's effectiveness may be from exercising several processes such as selective attention, sustained attention, and response inhibition. Further, in three neglect patients, TAPAT training produced larger behavioral improvements than a computerized search training task. Though preliminary, these results suggests that TAPAT-related improvements may not be due to simply engaging in a supervised training program for 9 days and that training non-spatial attention may be more effective for improving neglect symptoms than directly training spatial attention.

\section{ACKNOWLEDGMENTS}

We would like to acknowledge the efforts and dedication of the patients performing the testing and training, Albert Hoang-duc for his help in programming, data consolidation, and analysis, as well as Lynn Roberston, William Milberg, and Regina McGlinchey for fruitful discussions. This work was supported by VA Merit Grant \# 05-09-00371.

search: a large scale analysis. Cortex 40 , 282-290.

Bellgrove, M. A., Dockree, P. M., Aimola, L., and Robertson, I. H. (2004). Attenuation of spatial attentional asymmetries with poor sustained attention. Neuroreport 15, 1065-1069.

Beschin, N., and Robertson, I. H. (1997). Personal versus extrapersonal neglect: a group study of their dissociation using a reliable clinical test. Cortex 33 , 379-384.

Bisiach, E., Perani, D., Vallar, G., and Berti, A. (1986). Unilateral neglect: personal and extra-personal. Neuropsychologia 24, 759-767.

Boggio, P. S., Nunes, A., Rigonatti, S. P., Nitsche, M. A., Pascual-Leone, A., and Fregni, F. (2007). Repeated 
sessions of noninvasive brain DC stimulation is associated with motor function improvement in stroke patients. Restor. Neurol. Neurosci. 25, 123-129.

Bouret, S., and Sara, S. J. (2005). Network reset: a simplified overarching theory of locus coeruleus noradrenaline function. Trends Neurosci. 28, 574-582.

Brefczynski-Lewis, J.A., Lutz, A., Schaefer, H. S., Levinson, D. B., and Davidson, R. J. (2007). Neural correlates of attentional expertise in long-term meditation practitioners. Proc. Natl. Acad. Sci. U.S.A. 104, 11483.

Brighina, F., Bisiach, E., Oliveric, M. Piazza, A., La Bua, V., Daniele, O., and Fierro, B. (2003). $1 \mathrm{~Hz}$ repetitive transcranial magnetic stimulation of the unaffected hemisphere ameliorates contralesional visuospatial neglect in humans. Neurosci. Lett. 336, 131-133.

Buxbaum, L. J., Ferraro, M. K., Veramonti, T., Farne, A., Whyte, J., Ladavas, E., Frassinetti, F., and Coslett, H. B. (2004). Hemispatial neglect: subtypes, neuroanatomy, and disability. Neurology 62, 749-756.

Corbetta, M., Kincade, M. J., Lewis, C., Snyder, A. Z., and Sapir, A. (2005). Neural basis and recovery of spatial attention deficits in spatial neglect. Nat. Neurosci. 8, 1603-1610.

Coull, J. T., Nobre, A. C., and Frith, C. D. (2001). The noradrenergic alpha2 agonist clonidine modulates behavioural and neuroanatomical correlates of human attentional orienting and alerting. Cereb Cortex. 11, 73-84.

Draganski, B., and May, A. (2008). Training-induced structural changes in the adult human brain. Behav. Brain Res. 192, 137

Fink, G. R., Marshall, J. C., Weiss, P. H., Toni, I., and Zilles, K. (2002). Task instructions influence the cognitive strategies involved in line bisection judgements: evidence from modulated neural mechanisms revealed by fMRI. Neuropsychologia 40, 119-130.

Halligan, P.W., and Marshall, J. C. (1991). Left neglect for near but not far space in man. Nature 350, 498-500.

Harvey, M., Milner, A. D., and Roberts, R. C. (1995). An investigation of hemispatial neglect using the Landmark Task. Brain Cogn. 27, 59-78.

Heilman, K. M., Schwartz, H. D., and Watson, R. T. (1978). Hypoarousal in patients with the neglect syndrome and emotional indifference. Neurology 28, 229-232.

Hjaltason, H., Tegner, R., Tham, K., Levander, M., and Ericson, K. (1996).
Sustained attention and awareness of disability in chronic neglect. Neuropsychologia 34, 1229-1233.

Husain, M., and Rorden, C. (2003). Nonspatially lateralized mechanisms in hemispatial neglect. Nat. Rev. Neurosci. 4, 26-36.

Husain, M., Shapiro, K., Martin, J., and Kennard, C. (1997). Abnormal temporal dynamics of visual attention in spatial neglect patients. Nature 385, 154-156.

Kaernbach, C. (1990). A single-interval adjustment-matrix (SIAM) procedure for unbiased adaptive testing. J. Acoust. Soc. Am. 88, 2645-2655.

Katz, N., Hartman-Maeir, A., Ring, H., and Soroker, N. (1999). Functional disability and rehabilitation outcome in right hemisphere damaged patients with and without unilateral spatial neglect. Arch. Phys. Med. Rehabil. 80, 379-384.

Kranczioch, C., Debener, S., Schwarzbach, J., Goebel, R., and Engel, A. K. (2005) Neural correlates of conscious perception in the attentional blink. Neuroimage 24, 704-714.

Lazar, R. M., Fitzsimmons, B. F., Marshall, R. S., Berman, M. F., Bustillo, M. A., Young, W. L., Mohr, J. P., Shah, J., and Robinson, J.V.(2002). Reemergence of stroke deficits with midazolam challenge. Stroke 33, 283-285.

List, A., Brooks, J. L., Esterman, M. Flevaris, A. V., Landau, A. N., Bowman, G., Stanton, V., Vanvleet, T. M., Robertson, L. C., and Schendel, K. (2008). Visual hemispatial neglect, re-assessed. J. Int. Neuropsychol. Soc. 14, 243-256.

Luaute, J., Halligan, P., Rode, G., Rossetti, Y., and Boisson, D. (2006). Visuospatial neglect: a systematic review of current interventions and their effectiveness. Neurosci. Biobehav. Rev 30, 961-982.

Malhotra, P., Coulthard, E. J., and Husain, M. (2009). Role of right posterior parietal cortex in maintaining attention to spatial locations over time. Brain 132, 645-660.

Manly, T., Dobler, V. B., Dodds, C. M. and George, M. A. (2005). Rightward shift in spatial awareness with declining alertness. Neuropsychologia 43, 1721-1728.

McGlinchey-Berroth, R., Bullis, D. P., Milberg, W. P., Verfaellie, M., Alexander, M., and D'Esposito, M. (1996). Assessment of neglect reveals dissociable behavioral but not neuroanatomical subtypes. J. Int. Neuropsychol. Soc. 2, 441-451.

Naeser, M. A., Martin, P. I., Nicholas, M., Baker, E. H., Seekins, H., Kobayashi, M., Theoret, H., Fregni, F., Maria-
Tormos, J., Kurland, J., Doron, K. W., and Pascual-Leone, A. (2005) Improved picture naming in chronic aphasia after TMS to part of right Broca's area: an open-protocol study. Brain Lang. 93, 95-105.

Nieuwenhuis, S., Aston-Jones, G. and Cohen, J. D. (2005). Decision making, the P3, and the locus coeruleusnorepinephrine system. Psychol. Bull. 131, 510-532.

Olk, B., Wee, J., and Kingstone, A. (2004) The effect of hemispatial neglect on the perception of centre. Brain Cogn 55, 365-367.

Ota, H., Fijii, T., Suzuki, K., Fukatsu, R., and Yamadori, A. (2001). Dissociation of body-centered and stimuluscentered representations in unilateral neglect. Neurology 57, 2064.

Pizzamiglio, L., Guariglia, C., Antonucci, G., and Zoccolotti, P. (2006) Development of a rehabilitative program for unilateral neglect. Restor. Neurol. Neurosci. 24, 337-345.

Posner, M. I. (2008). Measuring Alertness. Ann. N. Y. Acad. Sci. 1129 193-199.

Raymond, J. E., Shapiro, K. L., and Arnell, K. M. (1992). Temporary suppression of visual processing in an RSVP task: an attentional blink? J. Exp. Psychol. Hum. Percept. Perform. 18, 849-860.

Rensink, R. A. (2002). Change detection. Annu. Rev. Psycholol. 53, 245-277.

Robertson, I. H. (2001). Do we need the "lateral" in unilateral neglect? Spatially nonselective attention deficits in unilateral neglect and their implications for rehabilitation. Neuroimage 14 S85-S90.

Robertson, I. H., Manly, T., Andrade, J. Baddeley, B. T., and Yiend, J. (1997a). 'Oops!': performance correlates of everyday attentional failures in traumatic brain injured and normal subjects. Neuropsychologia 35, 747-758.

Robertson, I. H., Manly, T., Beschin, N., Daini, R., Haeske-Dewick, H., Hömberg, V., Jehkonen, M. Pizzamiglio, G., Shiel,A., and Weber, E. (1997b). Auditory sustained attention is a marker of unilateral spatial neglect. Neuropsychologia 35, 1527-1532.

Robertson, I. H., Mattingley, J. B., Rorden, C., and Driver, J. (1998). Phasic alerting of neglect patients overcomes their spatial deficit in visual awareness. Nature 395, 169-172.

Robertson, I. H., Tegner, R., Tham, K. Lo, A., and Nimmo-Smith, I. (1995). Sustained attention training for unilateral neglect: theoretical and rehabilitation implications. J. Clin. Exp. Neuropsychol. 17, 416-430.

Roth, J. K., Serences, J. T., and Courtney, S. M. (2006). Neural system for con- trolling the contents of object working memory in humans. Cereb. Cortex 16 1595-1603.

Ryan, M., Martin, R., Denckla, M. B., Mostofsky, S. H., and Mahone, E. M. (2010). Interstimulus jitter facilitates response control in children with ADHD. J. Int. Neuropsychol. 16, 388-393.

Salthouse, T. A. (2000). Aging and measures of processing speed. Biol. Psychol. 54, 35-54.

Schulz, K. F., and Grimes, D. A. (2002) Generation of allocation sequences in randomised trials: chance, not choice. Lancet 359, 515-519.

Shapiro, K. L., Raymond, J. E., and Arnell, K. M. (1994). Attention to visual pattern information produces the attentional blink in rapid serial visual presentation. J. Exp. Psychol. Hum. Percept. Perform. 20, 357-371.

Smallwood, J., Davies, J. B., Heim, D., Finnigan, F., Sudberry, M., O'Connor, R., and Obonsawin, M. (2004). Subjective experience and the attentional lapse: task engagement and disengagement during sustained attention. Conscious. Cogn. 13, 657-690.

Sturm, W., de Simone, A. Krause, B. J., Specht, K., Hesselmann, V., Radermacher, I., Herzog, H., Tellmann, L., Müller-Gärtner, H. W., and Willmes, K. (1999). Functional anatomy of intrinsic alertness: evidence for a fronto-parietal-thalamic-brainstem network in the right hemisphere. Neuropsychologia 37, 797-805.

Sturm, W., Longoni, F., Weis, S., Specht, K., Herzog, H., Vohn, R., Thimm, M., and Willmes, K. (2004). Functional reorganisation in patients with right hemisphere stroke after training of alertness: a longitudinal PET and PMRI study in eight cases. Neuropsychologia 42, 434-450.

Sturm, W., and Willmes, K. (2001). On the functional neuroanatomy of intrinsic and phasic alertness. Neuroimage 14(Pt 2), S76-S84.

Tang, Y. Y., and Posner, M. I. (2009). Attention training and attention state training. Trends Cogn. Sci. (Regul.Ed.) 13, 222-227.

Thiel, C. M., Zilles, K., and Fink, G. R. (2004). Cerebral correlates of alerting, orienting and reorienting of visuospatial attention: an eventrelated fMRI study. Neuroimage 21, 318-328.

Thimm, M., Fink, G. R., Küst, J., Karbe, H., and Sturm, W. (2006). Impact of alertness training on spatial neglect: a behavioural and fMRI study. Neuropsychologia 44, 1230-1246. 
Toraldo, A., McIntosh, R. D., Dijkerman, H. C., and Milner, A. D. (2004). A revised method for analysing neglect using the landmark task. Cortex 40, 415-431.

Van Vleet, T. M., and Robertson, L. C. (2006). Cross-modal interactions in time and space: auditory influence on visual attention in hemispatial neglect. J. Cogn. Neurosci. 18, 1368-1379.
Wilcoxon, F. (1945). Individual comparisons by ranking methods. Biometrics 1, 80-83.

Young, A. W., Hellawell, D. J., and Welch, J. (1992). Neglect and visual recognition. Brain 115(Pt 1), 51-71.

Conflict of Interest Statement: The training procedure described in the current study is in the process of being patented (by the Department of Veterans Affairs) and licensed in order to develop it into a commercial training procedure.

Received: 15 April 2010; paper pending published: 25 May 2010; accepted: 12 July 2010; published online: 24 August 2010. Citation: DeGutis JM and Van Vleet T (2010) Tonic and phasic alertness training: $a$ novel behavioral therapy to improve spatial and non-spatial attention in patients with hemispatial neglect. Front. Hum. Neurosci. 4:60. doi: 10.3389/fnhum.2010.00060 Copyright $($ ) 2010DeGutisand Van Vleet. This is an open-access article subject to an exclusive license agreement between the authors and the Frontiers Research Foundation, which permits unrestricted use, distribution, and reproduction in any medium, provided the original authors and source are credited. 